\title{
A General Lee-Yang Theorem for One-Component and Multicomponent Ferromagnets
}

\author{
Elliott H. Lieb ${ }^{1 \star}$ and Alan D. Sokal ${ }^{2 \star \star}$ \\ 1 Departments of Mathematics and Physics, Princeton University, Princeton, NJ 08544, USA \\ 2 Department of Physics, Princeton University, Princeton, NJ 08544, USA
}

\begin{abstract}
We show that any measure on $\mathbb{R}^{n}$ possessing the Lee-Yang property retains that property when multiplied by a ferromagnetic pair interaction. Newman's Lee-Yang theorem for one-component ferromagnets with general single-spin measure is an immediate consequence. We also prove an analogous result for two-component ferromagnets. For $N$-component ferromagnets $(N \geqq 3$ ), we prove a Lee-Yang theorem when the interaction is sufficiently anisotropic.
\end{abstract}

\section{Introduction}

The Lee-Yang theorem on the zeros of the partition function is an important tool in the rigorous study of phase transitions in lattice spin systems [1]. In addition, it has applications to the proof of existence of the infinite-volume limit [2] and of a mass gap $[3,4]$, and to the proof of correlation inequalities $[5,6]$ and inequalities for critical exponents $[4,7,8]$.

In this paper we shall give a new proof of a generalized Lee-Yang Theorem. Our methods lead to an essentially complete result for one-component and two-component (classical) ferromagnets with quite general single-spin measures. We have also some promising partial results for $N$-component ferromagnets $(N \geqq 3)$. We end the paper with some conjectures.

Consider, for purposes of orientation, the model of one-component "spins" $\phi_{i}$ defined by the partition function

$$
Z=\int \exp \left[\sum_{i, j=1}^{n} J_{i j} \varphi_{i} \varphi_{j}+\sum_{i=1} h_{i} \varphi_{i}\right] \prod_{i=1}^{n} d v_{i}\left(\varphi_{i}\right) .
$$

Here the $d v_{i}$ are suitable probability measures on the real line; the pair interaction coefficients $J_{i j}$ are nonnegative ("ferromagnetic"); and the magnetic fields $h_{i}$ are allowed to take arbitrary complex values. The Lee-Yang theorem then states

$\star$ Research supported in part by NSF grant PHY 78-25390 A01

$\star \star$ Research supported in part by NSF grant PHY 78-23952 
that, for suitable measures $d v_{i}$, the partition function $Z\left(h_{1}, \ldots, h_{n}\right)$ is nonzero whenever $\operatorname{Re} h_{i}>0$ for all $i$. The theorem was originally proven by Lee and Yang [9] only for the spin- $\frac{1}{2}$ model

$$
d v_{i}(\varphi)=\frac{1}{2}[\delta(\varphi-1)+\delta(\varphi+1)] d \varphi, \text { all } i .
$$

Subsequently, numerous alternate proofs for the spin- $\frac{1}{2}$ case were found $[10-17,41]$, and the theorem was also extended to more general single-spin measures $d v_{i}[18,19,15,16,42,43]$. The best result is that of Newman [15], which allows arbitrary even measures $d v_{i}$ with the property that

$$
\int e^{h \varphi} d v_{i}(\varphi) \neq 0 \text { for } \operatorname{Re} h \neq 0 \text {, all } i \text {. }
$$

This result is essentially the best possible: it states that the Lee-Yang property holds for all $J_{i j} \geqq 0$ if and only if it holds for $J_{i j} \equiv 0$. But while the condition (1.3) is exceedingly natural, Newman's method of proof is quite indirect: he shows that (1.3) is a necessary and sufficient condition for the model (1.1) to be approximable in a certain sense by spin- $\frac{1}{2}$ models; and then he appeals to the already proven Lee-Yang theorem for the spin- $\frac{1}{2}$ case. The original motivation of the present work, therefore, was to find a direct proof of Newman's result, utilizing directly the condition (1.3). We did discover a rather elementary such proof; it is given in Appendix A. But we also discovered a far-reaching generalization of Newman's theorem, one which we believe clarifies the underlying structure of the Lee-Yang theorem.

Our method is based on the identity

$$
Z\left(h_{1}, \ldots, h_{n}\right)=\exp \left[\sum_{i, j=1}^{n} J_{i j} \frac{\partial}{\partial h_{j}} \frac{\partial}{\partial h_{j}}\right] Z_{0}\left(h_{1}, \ldots, h_{n}\right)
$$

where

$$
Z_{0}\left(h_{1}, \ldots, h_{n}\right)=\int \exp \left[\sum_{i=1}^{n} h_{i} \varphi_{i}\right] \prod_{i=1}^{n} d v_{i}\left(\varphi_{i}\right) .
$$

Now the hypothesis (1.3) ensures precisely that $Z_{0}$ has the Lee-Yang property; so what we need to show is that this property is preserved by a certain (infiniteorder) differential operator. Noting additionally the identity

$$
\exp \left[\sum_{i, j=1}^{n} J_{i j} z_{i} z_{j}\right]=\lim _{k \rightarrow \infty} \prod_{i, j=1}^{n}\left(1+k^{-1} J_{i j} z_{i} z_{j}\right)^{k},
$$

and taking account of the hypothesis $J_{i j} \geqq 0$, the Lee-Yang theorem is then reduced (modulo the approximation of entire functions by polynomials) to the following proposition about polynomials: if $P\left(z_{1}, \ldots, z_{n}\right)$ and $Q\left(z_{1}, \ldots, z_{n}\right)$ are polynomials which are nonvanishing when $\operatorname{Re} z_{i}>0$ for all $i$, then the polynomial $S\left(z_{1}, \ldots, z_{n}\right)=P\left(\partial / \partial z_{1}, \ldots, \partial / \partial z_{n}\right) Q\left(z_{1}, \ldots, z_{n}\right)$ also has this property (or else is identically zero). Now this is a well-known result in the case $n=1$ (Proposition 2.1); but it is also true in general, as we demonstrate (Proposition 2.2).

In fact, we deduce immediately the following generalization of Newman's result: Let $d \mu_{0}$ be any measure on $\mathbb{R}^{n}$ (not necessarily a product measure) posses- 
sing the "Lee-Yang property" (defined precisely in Sect. 3); then, for any set of $J_{i j} \geqq 0$, the measure

$$
d \mu(\varphi)=\exp \left[\sum_{i, j=1}^{n} J_{i j} \varphi_{i} \varphi_{j}\right] d \mu_{0}(\varphi)
$$

also has the Lee-Yang property. In other words, ferromagnetic pair interactions (among others) are "universal multipliers for Lee-Yang measures".

Similar considerations yield a gemeral Lee-Yang theorem for two-component ferromagnets: Let

$$
Z=\int \exp \left[\sum_{i, j=1}^{n} \sum_{\alpha=1}^{2} J_{i j}^{(\alpha)} \varphi_{i}^{(\alpha)} \varphi_{j}^{(\alpha)}+\sum_{i=1}^{n} \sum_{\alpha=1}^{2} h_{i}^{(\alpha)} \varphi_{i}^{(\alpha)}\right] \prod_{i=1}^{n} d v_{i}\left(\varphi_{i}\right),
$$

where $J_{i j}^{(1)} \geqq\left|J_{i j}^{(2)}\right|$ for all $i, j$, and each $d v_{i}$ is a rotationally symmetric measure on $\mathbb{R}^{2}$ whose projection onto one of the coordinates has the Lee-Yang property. Then $Z \neq 0$ whenever $\operatorname{Re} h_{i}^{(1)}>\left|\operatorname{Im} h_{i}^{(2)}\right|$ for all $i$. This generalizes a result obtained by Dunlop [20] for the plane-rotator model

$$
d v_{i}(\varphi)=\delta\left(|\varphi|^{2}-1\right) d \varphi, \text { all } i
$$

by infinitely more complicated (though intriguing) methods.

Sadly, we are unable to give a similarly complete solution of the Lee-Yang problem for $N$-component ferromagnets with $N \geqq 3$. At present, we have only the following partial result: in the obvious generalization of (1.8), one has $Z \neq 0$ whenever

$$
\operatorname{Re} h_{i}^{(1)}>\left[\sum_{\alpha=2}^{N}\left(\operatorname{Im} h_{i}^{(\alpha)}\right)^{2}\right]^{1 / 2} \text { for all } i
$$

provided that

$$
J_{i j}^{(1)} \geqq \sum_{\alpha=2}^{N}\left|J_{i j}^{(\alpha)}\right| \text { for all } i, j .
$$

This is a Lee--Yang theorem for highly anisotropic $N$-component ferromagnets, the first such result (known to us) for $N>3$. On the other hand, it is clearly unsatisfactory: the condition (1.11) ought to be replaced by

$$
J_{i j}^{(1)} \geqq \max _{2 \leqq \alpha \leqq N}\left|J_{i j}^{(\alpha)}\right| \text { for all } i, j,
$$

as is known by entirely different methods $[10,11,21,20]$ for $N=3$ (with a restricted class of single-spin measures). This result (for all $N$ ) would indeed follow by an extension of our methods, as we indicate in Sect. 5 , provided that an as-yetunproven generalization of Proposition 2.2 is true. But we are unable to find a proof - we hope that others will be more clever!

\section{General Theorems}

We begin with a result about polynomials of a single complex variable, which gives the flavor of our methods. 
Proposition 2.1. Let $P$ and $Q$ be polynomials in a single complex variable, with the property that $P(z) \neq 0$ whenever $\operatorname{Re} z>0$, and $Q(z) \neq 0$ whenever $\operatorname{Re} z>c$ (c real). Then $S(z) \equiv P(d / d z) Q(z)$ is either nonvanishing whenever $\operatorname{Re} z>c$ or else is identically zero. Moreover, $S(z) \equiv 0$ if and only if $P(z)$ has a zero at $z=0$ of order $m>\operatorname{deg} Q$.

Proof. $P$ can be factored as

$$
P(z)=a \prod_{i=1}^{\operatorname{deg} P}\left(z-\alpha_{i}\right)
$$

with $a \neq 0$ and $\operatorname{Re} \alpha_{i} \leqq 0$ for all $i$. Hence it suffices to prove the proposition for $P(z)=z-\alpha, \operatorname{Re} \alpha \leqq 0$; the general case follows by repeated application of this special case. Now $Q$ can be factored as

$$
Q(z)=b \prod_{j=1}^{\operatorname{deg} Q}\left(z-\beta_{j}\right)
$$

with $b \neq 0$ and $\operatorname{Re} \beta_{j} \leqq c$ for all $j$. Then

$$
\frac{Q^{\prime}(z)}{Q(z)}=\sum_{j=1}^{\operatorname{deg} Q} \frac{1}{z-\beta_{j}},
$$

and this has strictly positive real part whenever $\operatorname{Re} z>c$ (unless $\operatorname{deg} Q=0$, in which case it is identically zero). Hence $Q^{\prime}(z) / Q(z) \neq \alpha$ whenever $\operatorname{Re} z>c$ (unless $\operatorname{deg} Q=0$ and $\alpha=0)$; that is, $P(d / d z) Q(z)=Q^{\prime}(z)-\alpha Q(z) \neq 0$ for $\operatorname{Re} z>c$. The last assertion of the proposition is easily verified. QED.

Remarks. 1. Proposition 2.1 is actually a special case of a much more general result of Takagi [22] (see Marden [23, pp. 82-84]). The proof given here is a simplification of the method of Benz [32]; it is modeled on the standard proof of the Gauss-Lucas theorem [23, p. 22].

2. The arbitrariness of $c$ is a trivial consequence of invariance under translation of the variable associated with $Q$. Note, however, that the variable associated with $P$ cannot be translated; here zero is a distinguished point.

3. Proposition 2.1 was implicitly noted by Newman [15] in the course of the proof of an intermediate result (his Proposition 2.4). It was our attempt to understand the role of this proposition in the proof of the Lee-Yang theorem that led to the present work.

Proposition 2.1 is already sufficient, together with the approximation theorems given later in this section, to prove Newman's version of the Lee-Yang theorem; this proof is given in Appendix A. But it is in fact possible to prove a yet more general result which makes clear (we believe) what is really going on in the LeeYang theorem. To do this, we need a multi-variable generalization of Proposition 2.1.

Notation. If $z=\left(z_{1}, \ldots, z_{n}\right) \in \mathbb{C}^{n}$ and $c=\left(c_{1}, \ldots, c_{n}\right) \in \mathbb{R}^{n}$, then $\operatorname{Re} z>c$ means that $\operatorname{Re} z_{j}>c_{j}$ for all $j$; analogously for $\operatorname{Re} z \geqq c$. $\partial / \partial z$ means the $n$-tuple $\left(\partial / \partial z_{1}, \ldots\right.$, $\left.\partial / \partial z_{n}\right)$. 
Proposition 2.2. Let $P_{i}$ and $Q_{i}(1 \leqq i \leqq k)$ be polynomials in $n$ complex variables, and define

$$
R(v, w)=\sum_{i=1}^{k} P_{i}(v) Q_{i}(w)
$$

and

$$
S(z)=\sum_{i=1}^{k} P_{i}(\partial / \partial z) Q_{i}(z) .
$$

(a) If $R(v, w) \neq 0$ whenever $\operatorname{Re} v \geqq 0$ and $\operatorname{Re} w \geqq c\left(c \in \mathbb{R}^{n}\right)$, then $S(z) \neq 0$ whenever $\operatorname{Re} z \geqq c$.

(b) If $R(v, w) \neq 0$ whenever $\operatorname{Re} v>0$ and $\operatorname{Re} w>c$, then either $S(z) \neq 0$ whenever $\operatorname{Re} z>c$, or else $S(z)$ is identically zero.

This Theorem generalizes Proposition 2.1 in two major ways: first, the single complex variable is replaced by $n$ complex variables; and second, the single product $P Q$ is replaced by a sum of $k$ such terms. The second generalization can be thought about as follows: write $R(v, w)$ as a sum of monomials with all variables $v$ standing to the left of all variables $w$; then $S(z)$ is obtained by replacing each $v_{j}(1 \leqq j \leqq n)$ by $\partial / \partial z_{j}$, and each $w_{j}$ by $z_{j}$. This representation makes clear that $S$ depends only on $R$, not on the particular decomposition of $R$ into $\sum P_{i} Q_{i}$. This second generalization is of no particular interest for the application we have in mind, but it turns our to be quite natural for the proof of the propositions.

Note first that by translation invariance (Remark 2 above), we can take $c=0$. We then proceed in a series of lemmas:

Lemma 2.3. Let $Q_{0}$ and $Q_{1}$ be polynomials in a single complex variable, and assume that $R(v, w) \equiv Q_{0}(w)+v Q_{1}(w) \neq 0$ whenever $\operatorname{Re} v \geqq 0$ and $\operatorname{Re} w \geqq 0$. Then $S(z) \equiv$ $Q_{0}(z)+Q_{1}^{\prime}(z) \neq 0$ whenever $\operatorname{Re} z \geqq 0$.

Proof. Setting $v=0$, we find that $Q_{0}(z) \neq 0$ whenever $\operatorname{Re} z \geqq 0$. If $Q_{1} \equiv 0$, this completes the proof; so assume that $Q_{1} \not \equiv 0$. Then, letting $c \rightarrow+\infty$, we find that $Q_{1}(z) \neq 0$ whenever $\operatorname{Re} z>0$ (for otherwise, by Hurtwitz' Theorem [23, p. 4] applied to $v^{-1} Q_{0}+Q_{1}$, there would exist zeros of $R(v, w)$ with $\operatorname{Re} w>0$ for any sufficiently large $|v|)$. Moreover, if $Q_{1}\left(z_{0}\right)=0$ and $\operatorname{Re} z_{0}=0$, then $Q_{1}^{\prime}\left(z_{0}\right) / Q_{0}\left(z_{0}\right)$ is real and nonnegative (for otherwise, by the implicit function theorem, there would exists zeros of $R(v, w)$ with $w$ near $z_{0}$ and $\operatorname{Re} w>0$ for suitable (large) $v$ with $\operatorname{Re} v>0$ ). Finally, we note that $\operatorname{Re}\left[Q_{0}(z) / Q_{1}(z)\right]>0$ whenever $\operatorname{Re} z \geqq 0$ and $Q_{1}(z) \neq 0$ (for otherwise there would exist a zero of $R(v, w)$ with $\operatorname{Re} v \geqq 0$ and $\operatorname{Re} w \geqq 0$ ).

Now $Q_{1}$ can be factored as

$$
Q_{1}(z)=b \prod_{j=1}^{\operatorname{deg} Q_{1}}\left(z-\beta_{j}\right)
$$

with $b \neq 0$ and $\operatorname{Re} \beta_{j} \leqq 0$ for all $j$. Then

$$
\frac{Q_{1}^{\prime}(z)}{Q_{1}(z)}=\sum_{j=1}^{\operatorname{deg} Q_{1}} \frac{1}{z-\beta_{j}},
$$


and this has nonnegative real part for $\operatorname{Re} z \geqq 0$ (except at the zeros of $Q_{1}$, where it is undefined). Hence

$$
Q_{0}(z)+Q_{1}^{\prime}(z)=Q_{1}(z)\left[\frac{Q_{0}(z)}{Q_{1}(z)}+\frac{Q_{1}^{\prime}(z)}{Q_{1}(z)}\right] \neq 0
$$

whenever $\operatorname{Re} z \geqq 0$ and $Q_{1}(z) \neq 0$. On the other hand, if $\operatorname{Re} z=0$ and $Q_{1}(z)=0$, then

$$
Q_{0}(z)+Q_{1}^{\prime}(z)=Q_{0}(z)\left[1+\frac{Q_{1}^{\prime}(z)}{Q_{0}(z)}\right] \neq 0 .
$$

This completes the proof.

Remark 1. It is indeed possible for $Q_{1}$ to have zeros on the imaginary axis: consider, for example, $Q_{0}(w)=1+w$ and $Q_{1}(w)=w$.

Remark 2. A related result has been obtained by Dieudonne [24].

Lemma 2.4. (Grace [25]). Let $K \subset \mathbb{C}$ be a circular region (i.e. a closed disc, the closed exterior of a disc, or a closed half-plane), and let

$$
F(x)=\sum_{m=0}^{N} a_{m} x^{m}
$$

be a polynomial which is nonvanishing whenever $x \in K$. Next let $x_{1}, \ldots, x_{N}$ be complex variables, and let $E_{0}, \ldots, E_{N}$ be the elementary symmetric functions of the $\left\{x_{i}\right\}$, i.e. $E_{0}=1, E_{1}=\sum_{i} x_{i}$, and

$$
E_{m}=\sum_{i_{1}<i_{2}<\ldots<i_{m}} x_{i_{1}} x_{i_{2}} \ldots x_{i_{m}}
$$

Then the polynomial

$$
\tilde{F}\left(x_{1}, \ldots, x_{N}\right)=\sum_{m=0}^{N} a_{m}\left(\begin{array}{l}
N \\
m
\end{array}\right)^{-1} E_{m}\left(x_{1}, \ldots, x_{N}\right)
$$

is nonvanishing whenever $x_{1}, \ldots, x_{N}$ are all in $K$.

Proof. See Obreschkoff [26, pp. 23-24] or Marden [23, pp. 62-63].

Proof of Proposition 2.2. Let $N$ be any integer $\geqq$ the maximal degree of $R(v, w)$ in any of the variables $v_{j}$; and introduce new variables $v_{j}^{(k)}, 1 \leqq k \leqq N$. Now let $\tilde{R}(V, w)$ be the polynomial obtained by expanding $R(v, w)$ as a sum of monimials and replacing each factor $v_{j}^{m}$ by

$$
\left(\begin{array}{l}
N \\
m
\end{array}\right)^{-1} E_{m}\left(v_{j}^{(1)}, \ldots, v_{j}^{(N)}\right) .
$$

By repeated application of Lemma $2.4, \tilde{R}(V, w)$ is nonvanishing whenever $\operatorname{Re} v_{j}^{(k)} \geqq 0$ for all $j, k$ and $\operatorname{Re} w_{j} \geqq 0$ for all $j$. Now $\tilde{R}(V, w)$ is of degree at most 1 in each variable $v_{j}^{(k)}$; so we can repeatedly apply Lemma 2.3 to convert each $v_{j}^{(k)}$ into $\partial / \partial w_{j}$, while all other variables are fixed in the closed right half-plane. The result of this process is easily seen to be $S(w)$. This proves $(a)$. 
To prove $(b)$, let $\varepsilon>0$ and define $P_{i}^{(\varepsilon)}(v)=P_{i}\left(v_{1}+\varepsilon, \ldots, v_{n}+\varepsilon\right)$, and likewise for $Q_{i}^{(\varepsilon)}$; and define $R^{(\varepsilon)}$ and $S^{(\varepsilon)}$ in the obvious way involving $P_{i}^{(\varepsilon)}$ and $Q_{i}^{(\varepsilon)}$. Now clearly $R^{(\varepsilon)}(v, w) \neq 0$ whenever $\operatorname{Re} z \geqq 0$ and $\operatorname{Re} w \geqq 0$, so by part (a), $S^{(\varepsilon)}(z) \neq 0$ whenever $\operatorname{Re} z \geqq 0$. But $S^{(\varepsilon)}(z)$ converges to $S(z)$ as $\varepsilon \downarrow 0$, uniformly on compacts; so Hurwitz' theorem on $\mathbb{C}^{n}$ implies that either $S(z)$ is nonvanishing on the open set $\operatorname{Re} z>0$, or else $S(z)$ is identically zero. This completes the proof.

Remarks. 1. Part (b) of Proposition 2.2 can also be proven by an "elementary" argument (i.e. one avoiding Grace's Theorem); the proof is based on the identity

$$
S(w)=\left.\left[\prod_{j=1}^{n} \exp \left(\frac{\partial}{\partial v_{j}} \frac{\partial}{\partial w_{j}}\right)\right] R(v, w)\right|_{v=0} .
$$

More precisely, we define $R^{(\varepsilon)}$ as above, and note that

$$
S(w)=\lim _{\varepsilon \downarrow 0} \lim _{m \rightarrow \infty}\left(\left.\left[\prod_{j=1}^{n}\left(1+m^{-1}\left(\frac{\partial}{\partial v_{j}}+\varepsilon\right)\left(\frac{\partial}{\partial w_{j}}+\varepsilon\right)\right)^{m}\right] R^{(\varepsilon)}(v, w)\right|_{v=0}\right)
$$

uniformly on compacts. Now the differential operator in brackets is a product of polynomials each of which is of degree 1 in each variable and which is nonvanishing when the real parts of all variables are nonnegative; so it follows from Lemma 2.3, by a repetitive argument similar to that used above, that this operator preserves the nonvanishing of $R^{(\varepsilon)}$ for $\operatorname{Re} v \geqq 0, \operatorname{Re} w \geqq 0$. The conclusion of part (b) then follows by Hurwitz' theorem.

2. If the coefficients in $P_{i}$ and $Q_{i}$ are allowed to depend analytically on an auxiliary variable $\zeta$ varying in a domain $D \subset \mathbb{C}^{r}$ and the hypothesis of the proposition holds for all $\zeta \in D$, then in part (b) of the proposition, $S(z ; \zeta)$ can vanish identically for one value of $\zeta$ only if it does so for all $\zeta \in D$. This is an immediate consequence of including the variable $\zeta$ in the Hurwitz argument.

3. Grace's theorem has been employed in a similar way by Millard and Viswanathan [27].

Our next goal is to extend Propositions 2.1 and 2.2 to suitable classes of entire functions. If $f$ is an entire function $\mathbb{C}^{n}$, and $b>0$, we define

$$
\|f\|_{b}=\sup _{z \in \mathbb{C}}\left[\exp \left(-b \sum_{i=1}^{n}\left|z_{i}\right|^{2}\right)|f(z)|\right] \text {. }
$$

Then, for each $a \geqq 0$, let $\mathscr{A}_{a+}^{n}$ be the space of entire functions $f$ such that $\|f\|_{b}<\infty$ for all $b>a$. That is, $\mathscr{A}_{a+}^{n}$ is the space of entire functions of exponential order strictly less than 2 , or of order 2 and type at most $a$. We equip $\mathscr{A}_{a+}^{n}$ with the family of norms $\|\cdot\|_{b}, b>a$ (or equivalently, the countable family $\|\cdot\|_{a+1 / k}^{+}, k$ integer); then $\mathscr{A}_{a+}^{n}$ is a Fréchet space. Note also that $\mathscr{A}_{a+}^{n}$ is closed under differentiation; this is a simple consequence of the Cauchy integral formula. Finally, we note two other elementary facts about $\mathscr{A}_{a+}^{n}$ [28]:

1. A bounded sequence (or net) in $\mathscr{A}_{a+}^{n}$ converges in the topology of $\mathscr{A}_{a+}^{n}$ if and only if it converges pointwise on $\mathbb{C}^{n}$ (or even on an arbitrarily small nonempty open subset of $\mathbb{C}^{n}$ ).

2. For any $f \in \mathscr{A}_{a+}^{n}$, the partial sums of the Taylor series of $f$ converge to $f$ in the topology of $\mathscr{A}_{a+}^{n}$. Hence the polynomials are dense in $\mathscr{A}_{a+}^{n}$. 


$$
\text { Let } f(z)=\sum_{\mathbf{m}} \alpha_{\mathbf{m}} z^{\mathbf{m}} \text { and } g(z)=\sum_{\mathbf{m}} \beta_{\mathbf{m}} z^{\mathbf{m}}
$$

be entire functions on $\mathbb{C}^{n}$; then we can define the formal power series

$$
[f(\partial) g](z)=\sum_{\mathbf{k}} \sum_{\mathbf{m}} \alpha_{\mathbf{k}} \beta_{\mathbf{m}}\left(\partial / \partial z^{\mathbf{k}}\right) z^{\mathbf{m}}
$$

[Here $\mathbf{m}=\left(m_{1}, \ldots, m_{n}\right)$ is a multi-index, $z^{\mathbf{m}}=\prod_{i=1}^{n} z_{i}^{m_{i}}$ and $\partial / \partial z^{\mathbf{k}}=\partial / \partial z_{1}^{k_{1}} \ldots \partial z_{n}^{k_{n}}$. For suitable $f$ and $g$, we can actually make sense of (2.2):

Proposition 2.5. Let $a, b>0$ with $a b<\frac{1}{4}$, and let $c>b /(1-4 a b)$. Let $f, g$ be entire functions on $\mathbb{C}^{n}$ with $\|f\|_{a}<\infty,\|g\|_{b}<\infty$. Then the series (2.2) is absolutely convergent for all $z$, and defines an entire function such that

$$
\|f(\partial) g\|_{c} \leqq K_{a b c}^{n}\|f\|_{a}\|g\|_{b}
$$

for some $K_{a b c}^{n}<\infty$ independent off and $g$. It follows that

$$
(f, g) \mapsto f(\partial) g
$$

is a continuous bilinear map from $\mathscr{A}_{a+}^{n} \times \mathscr{A}_{b+}^{n}$ into $\mathscr{A}_{b /(1-4 a b)+}^{n}$, for any $a, b \geqq 0$ with $a b<\frac{1}{4}$.

Proof. By a simple estimate using the Cauchy integral formula,

$$
\begin{aligned}
& \left|\alpha_{\mathbf{k}}\right| \leqq\|f\|_{a} \prod_{i=1}^{n}\left(2 e a / k_{i}\right)^{k_{i} / 2} \\
& \left|\beta_{\mathbf{m}}\right| \leqq\|g\|_{b} \prod_{i=1}^{n}\left(2 e b / m_{i}\right)^{m_{\imath} / 2}
\end{aligned}
$$

(with $\left.0^{0} \equiv 1\right)$. Since $(k / 2 e)^{k / 2} \geqq C_{1} \Gamma((k+1) / 2)$ with $C_{1}>0$, it follows that

$$
\begin{aligned}
& \left|\alpha_{\mathbf{k}}\right| \leqq C_{2}\|f\|_{a} \prod_{i=1}^{n} a^{k_{i} / 2} / A\left(k_{i}\right) \\
& \left|\beta_{\mathbf{m}}\right| \leqq C_{2}\|g\|_{b} \prod_{i=1}^{n} b^{m_{i} / 2} / A\left(m_{i}\right)
\end{aligned}
$$

where we have defined $A(0)=1, A(2 s+1)=A(2 s+2)=s$ ! for $s=0,1,2, \ldots$. Therefore the proposition reduces to the case $n=1$ with

$$
\begin{aligned}
& f(z)=1+\left(z+z^{2}\right) \exp \left(a z^{2}\right) \\
& g(z)=1+\left(z+z^{2}\right) \exp \left(b z^{2}\right)
\end{aligned}
$$

and $z$ real and positive. Clearly the terms 1 are unimportant. The evaluation of the double series (2.2) is then a combinatorial problem that can be handled as follows: For $x$ real, write

$$
\exp \left(a x^{2}\right)=C_{3}(a) \int_{-\infty}^{\infty} \exp \left(-t^{2} / a+2 t x\right) d t
$$

and use this (formally) with $x=\partial / \partial z$. Since

$$
\exp (2 t \partial / \partial z) g(z)=g(z+2 t)
$$


we have

$$
\begin{aligned}
& \exp \left(a \partial^{2} / \partial z^{2}\right)\left[\left(z+z^{2}\right) \exp \left(b z^{2}\right)\right] \\
& \quad=C_{3}(a) \int_{-\infty}^{\infty}\left[(z+2 t)+(z+2 t)^{2}\right] \exp \left[-t^{2} / a+b(z+2 t)^{2}\right] d t \\
& \quad=P_{1}(a, b ; z) \exp \left(c^{\prime} z^{2}\right)
\end{aligned}
$$

where $c^{\prime}=b /(1-4 a b)$ and $P_{1}$ is a quadratic polynomial in $z$ whose coefficients depend only on $a$ and $b$. Then

$$
\begin{aligned}
& \left(\partial / \partial z+\partial^{2} / \partial z^{2}\right) \exp \left(a \partial^{2} / \partial z^{2}\right)\left[\left(z+z^{2}\right) \exp \left(b z^{2}\right)\right] \\
& \quad=P_{2}(a, b ; z) \exp \left(c^{\prime} z^{2}\right)
\end{aligned}
$$

where $P_{2}$ is a quartic polynomial in $z$ whose coefficients depend only on $a$ and $b$. The rest of the proof is easy.

Remarks. 1. A partially alternate proof can be based on the methods of [29, Theorem 7] or [30, Lemma 14.1.1.].

2. We do not know whether the estimate (2.3) is true for $c=b /(1-4 a b)$. We suspect that it is not, but we have no counterexample.

For any set $A \subset \mathbb{C}^{n}$, let $\mathscr{P}^{n}(A)$ be the set of polynomials on $\mathbb{C}^{n}$ which are nonvanishing on $A$ and let $\overline{\mathscr{P}_{a+}^{n}}(A)$ be the closure of $\mathscr{P}^{n}(A)$ in $\mathscr{A}_{a+}^{n}$. It follows immediately by Hurwitz' theorem that any $f \in \mathscr{P}_{\alpha+}^{n}(A)$ is either identically zero or else is nonvanishing in the interior of $A$. However, the converse is not true: as we shall see shortly (Proposition 2.7), there exist entire functions $f \in \mathscr{A}_{a+}^{n}$, nonvanishing on $A$, which are not approximable by polynomials nonvanishing on $A$.

Let $D^{n}$ denote the set $\left\{z \in \mathbb{C}^{n}: \operatorname{Re} z>0\right\}$. Then Propositions 2.2 and 2.5 immediately imply:

Proposition 2.6. Let $a, b \geqq 0$ with $a b<\frac{1}{4}$, and let $f \in \overline{\mathscr{P}_{a+}^{n}}\left(D^{n}\right)$ and $g \in \mathscr{P}_{b+}^{n}\left(D^{n}\right)$. Then $h(z)=f(\partial / \partial z) g(z)$ is in $\overline{\mathscr{P}_{b /(1-4 a b)+}^{n}}\left(D^{n}\right)$.

For a partial converse to Proposition 2.6, see [31,32] and [33, Sect. IX.6].

For our application we shall need to know which "pair interactions" lie in $\overline{\mathscr{P}_{a+}^{n}}\left(D^{n}\right)$. The criterion is simple:

Proposition 2.7. Let $B$ be a (complex) $n \times n$ symmetric matrix, and let $f(z)=\exp \left(\sum_{i, j} B_{i j} z_{i} z_{j}\right)$. Then the following are equivalent:

(a) $B_{i j} \geqq 0$ for all $i, j$.

(b) $f \in \overline{\mathscr{P}}_{\|B\|+}\left(D^{n}\right)$, where $\|B\|$ is the norm of $B$ considered as a bilinear form on $\mathbb{C}^{n}$ (or $\left.\mathbb{R}^{n}\right)$ equipped with the Euclidean norm.

(c) There exist polynomials $\left\{P_{i}\right\}$ in $\mathscr{P}^{n}\left(D^{n}\right)$ converging pointwise to $f$.

Proof. To prove (a) $\Rightarrow(\mathrm{b})$, note that

$$
f(z)=\lim _{k \rightarrow \infty} \prod_{i, j}\left(1+k^{-1} B_{i j} z_{i} z_{j}\right)^{k} ;
$$

and since $B_{i j} \geqq 0$, the polynomials on the right are all nonvanishing in $D^{n}$. More- 
over,

$$
\begin{aligned}
\left|\prod_{i, j}\left(1+k^{-1} B_{i j} z_{i} z_{j}\right)^{k}\right| & \leqq \exp \left(\sum_{i, j} B_{i j}\left|z_{i}\right| z_{j} \mid\right) \\
& \leqq \exp \left(\|B\| \sum_{i=1}^{n}\left|z_{i}\right|^{2}\right),
\end{aligned}
$$

from which the convergence in $\mathscr{A}_{\|B\|+}^{n}$ easily follows [28]. Clearly (b) implies (c). Finally, note that (c) implies that for fixed $\left(z_{2}, \ldots, z_{n}\right) \in D^{n-1}$, there exist polynomials $\left\{Q_{i}\right\}$ in $\mathscr{P}^{1}\left(D^{1}\right)$ converging pointwise to

$$
\widehat{f}(z)=\exp \left[B_{11} z^{2}+\left(2 \sum_{j=2}^{n} B_{1 j} z_{j}\right) z\right]
$$

Now $Q_{i} \in \mathscr{P}^{1}\left(D^{1}\right)$ implies that $\left|Q_{i}(z)\right| \geqq\left|Q_{i}\left(z^{\prime}\right)\right|$ whenever $\operatorname{Re} z \geqq\left|\operatorname{Re} z^{\prime}\right|$ and $\operatorname{Im} z=\operatorname{Im} z^{\prime}$, and this inequality clearly carries over to $\hat{f}$. But it is not hard to see that this implies $B_{11} \geqq 0$ and $\operatorname{Re} \sum_{j=2}^{n} B_{1 j} z_{j} \geqq 0$. Since this holds for all $\left(z_{2}, \ldots, z_{n}\right) \in$ $D^{n-1}$, we must have $B_{1 j} \geqq 0$ for $2 \leqq j \leqq n$. Analogously one shows that $B_{i j} \geqq 0$ for all $i, j$; hence (c) implies (a). QED.

Remarks. 1. $\|B\| \leqq \max _{i} \sum_{j}\left|B_{i j}\right|$, by a simple argument using the Riesz-Thorin interpolation theorem (or Hölder's inequality).

2. Polya [31] and Obrechkoff [34] have shown that $f \in \overline{\mathscr{P}}_{a^{+}}^{1}\left(D^{1}\right)$ if and only if

$$
f(z)=\mathrm{Ke}^{\beta z^{2}+\gamma z} \prod_{j}\left(1-\frac{z}{\alpha_{j}}\right) e^{a / \alpha_{j}}
$$

with $0 \leqq \beta \leqq a, \operatorname{Re} \alpha_{j} \leqq 0$ for all $j, \sum_{j}\left|\alpha_{j}\right|^{-2}<\infty$, and $\operatorname{Re} \gamma \geqq-\sum_{j} \operatorname{Re} \alpha_{j}^{-1}$. For a proof, see Levin [33, Sect. VIII.1]. Analogous results exist for various other regions in $\mathbb{C}^{1}[33,35]$.

For each $a>0$, let $\mathscr{T}_{a}^{n}$ be the space of tempered distributions $T$ on $\mathbb{R}^{n}$ such that

$$
T(x)=\exp \left[-a \sum_{i=1}^{n} x_{i}^{2}\right] T_{a}(x)
$$

for some tempered distribution $T_{a}$. We equip $\mathscr{T}_{a}^{n}$ with the weak topology generated by the test functions

$$
f(x)=\exp \left[a \sum_{i=1}^{n} x_{i}^{2}\right] f_{a}(x)
$$

with $f_{a} \in \mathscr{S}\left(\mathbb{R}^{n}\right)$. That is, a sequence (or net) of distributions $T^{(j)} \in \mathscr{T}_{a}^{n}$ converges to $T \in \mathscr{T}_{a}^{n}$ if and only if the distributions $T_{a}^{(j)}$ [defined as in (2.7)] converge to $T_{a}$ in the usual (weak) topology of $\mathscr{S}^{\prime}\left(\mathbb{R}^{n}\right)$. Also, we define $\mathscr{T}_{\infty}^{n}=\bigcap_{a>0} \mathscr{T}_{a}$, equipped with the obvious topology. 
Lemma 2.8. Let $0<a \leqq \infty$. Then the Laplace transform $T \mapsto \hat{T}$ defined $b y$

$$
\hat{T}(x)=\int e^{z \cdot x} T(x) d x
$$

is a sequentially continuous linear map of $\mathscr{T}_{a}^{n}$ into $\mathscr{A}_{1 / 4 a+}^{n}$. That is, if a sequence $T^{(j)}$ converges to $T$ in $\mathscr{T}_{a}^{n}$, then $\hat{T}^{(j)}$ converges to $\widehat{T}_{\text {in }} \mathscr{A}^{n}{ }_{1 / 4 a+}$.

Proof. Assume first that $0<a<\infty$. Then $\hat{T}(z)=T_{a}\left(f_{z}\right)$, where $f_{z}(x)=$ $\exp \left(z \cdot x-a x^{2}\right)$. Since $T_{a} \in \mathscr{S}^{\prime}\left(\mathbb{R}^{n}\right)$, we have.

$$
\left|T_{a}(f)\right| \leqq K \sup _{x}\left(1+|x|^{M}\right) \sum_{|\alpha| \leqq N}\left|\partial^{\alpha} f(x)\right|
$$

for some $K, M, N$. Now

$$
\sup _{x}\left(1+|x|^{M}\right) \sum_{|\alpha| \leqq N}\left|\partial^{\alpha} f_{z}(x)\right| \leqq C\left(1+|z|^{M+N}\right) \exp \left(|z|^{2} / 4 a\right)
$$

for a suitable constant $C$ (depending on $M, N, a$ ). Hence $T \in \mathscr{A}_{1 / 4 a+}^{n}$. Now fix $\beta>1 / 4 a$ and let $g_{z}(x)=\exp \left(z \cdot x-a x^{2}-\beta|z|^{2}\right)$. Then it follows from (2.11) that $\left\{g_{z}\right\}_{z \in \mathbb{C}^{n}}$ is a bounded family in $\mathscr{S}\left(\mathbb{R}^{n}\right)$. Hence, since weak and strong convergence are equivalent for sequences in $\mathscr{S}^{\prime}\left(\mathbb{R}^{n}\right)[36, \mathrm{pp} .74,238], T_{a}^{(j)}\left(g_{z}\right)$ converges to $T_{a}\left(g_{z}\right)$ uniformly for $z \in \mathbb{C}^{n}$; in other words, $\widehat{T}^{(j)}$ converges to $\hat{T}$ in $\mathscr{A}_{1 / 4 a+}^{n}$.

The case $a=\infty$ follows immediately from the foregoing, since convergence in $\mathscr{T}_{\infty}^{n}\left[\right.$ resp. $\left.\mathscr{A}_{0+}^{n}\right]$ is equivalent to convergence in $\mathscr{T}_{a}^{n}$ for all $a<\infty$ [resp. in $\mathscr{A}_{\varepsilon+}^{n}$ for all $\left.\varepsilon>0\right]$. QED.

Remark. We would get continuity instead of just sequential continuity if we had equipped $\mathscr{T}_{a}^{n}$ with the strong topology.

Proposition 2.9. Let $0 \leqq \alpha<\beta \leqq \infty$; let $T$ be a distribution in $\mathscr{T}_{\beta}^{n}$ whose Laplace transform $\hat{T}$ lies in $\overline{\mathscr{P}_{1 / 4 \beta+}^{n}}\left(D^{n}\right)$; and let $f \in \overline{\mathscr{P}_{\alpha+}^{n}}\left(D^{n}\right)$. Then, for every $\gamma<\beta-\alpha$ [and for $\gamma=\infty$ if $\beta=\infty$ ], the distribution $f$ T lies in $\mathscr{T}_{\gamma}^{n}$ and its Laplace transform $f$ T lies in $\overline{\mathscr{P}_{1 / 4 \gamma+}^{n}}\left(D^{n}\right)$.

Proof. Clearly $f T \in \mathscr{T}_{\gamma}^{n}$. To prove the statement about the Laplace transform, assume first that $f$ is a polynomial, i.e. $f \in \mathscr{P}^{n}\left(D^{n}\right)$. Then clearly

$$
\hat{f T}(z)=f(\partial / \partial z) \widehat{T}(z),
$$

so by Proposition 2.6 [with $a=\alpha, b=1 / 4 \beta$ ] we have

$\left.\widehat{f T} \in \overline{\mathscr{P}^{n}}\left(D^{n}\right) \subset \overline{\mathscr{P}^{n}} 1 / 4 \gamma+\alpha\right)+\left(D^{n}\right)$. For general $f \in \overline{\mathscr{P}_{\alpha+}^{n}}\left(D^{n}\right)$, let $\left\{f_{j}\right\}$ be a sequence in $\mathscr{P}^{n}\left(D^{n}\right)$. For general $f \in \mathscr{P}_{\alpha+}^{n}\left(D^{n}\right)$, let $\left\{f_{j}\right\}$ be a sequence in $\mathscr{P}^{n}\left(D^{n}\right)$ converging to $f$ in $\mathscr{A}_{a+}^{n}$. Then $\left\{f_{j} T\right\}$ converges to $f T$ in $\mathscr{T}_{\gamma}^{n}$, so by Lemma $2.8,\left\{\widehat{f_{j} T}\right\}$ converges to $f \widehat{T}$ in $\mathscr{A}_{1 / 4 \gamma+}^{n}$. Since $\widehat{\mathscr{P}}_{1 / 4 \gamma+}^{n}\left(D^{n}\right)$ is closed in $\mathscr{A}_{1 / 4 \gamma+}^{n}$, this proves the proposition.

Finally, let us append a remark which clarifies the "strong Lee-Yang theorem" of Newman [15, section 3]:

$$
\begin{aligned}
& \partial_{x}^{\mathbf{m}}|Z(x+i y)|^{2}>0 \text { whenever } x \in(0, \infty)^{n} \text { and } y \in \mathbb{R}^{n}, \\
& \quad \text { for every multi-index } \mathbf{m} .
\end{aligned}
$$

(Here we write $x=\operatorname{Re} h, y=\operatorname{Im} h$. The ordinary Lee-Yang theorem is just the 
case $\mathbf{m}=0$.) The point is that (2.12) is actually a consequence of our form of the Lee-Yang Theorem:

Proposition 2.10. Let $f$ be an analytic function on $D^{n}$ which is a limit, uniformly on compacts, of polynomials $P \in \mathscr{P}^{n}\left(D^{n}\right)$. [In particular, $f \in \overline{\mathscr{P}_{\alpha+}^{n}}\left(D^{n}\right)$ for some $\alpha$ suffices.] For $x \in(0, \infty)^{n}$ and $y \in \mathbb{R}^{n}$, let $F(x, y)=|f(x+i y)|^{2}$ and let $G_{\mathbf{m}}(x, y)=\partial^{\mathbf{m}} F / \partial x^{\mathbf{m}}$. Then, for each multi-index $\mathbf{m}$, we have either

(i) $G_{m}(x, y)>0$ for all $x \in(0, \infty)^{n}$ and all $y \in \mathbb{R}^{n}$ or else

(ii) $G_{\mathbf{m}}(x, y)=0$ for all $x \in(0, \infty)^{n}$ and all $y \in \mathbb{R}^{n}$.

Proof. Consider first the case $f=P \in \mathscr{P}^{n}\left(D^{n}\right)$. Let $Q(z)=\overline{P(\bar{z})}$; clearly $Q \in \mathscr{P}^{n}\left(D^{n}\right)$. Now define

$$
R\left(z, z^{\prime}\right)=P\left(z+i z^{\prime}\right) Q\left(z-i z^{\prime}\right) .
$$

Note that if $x$ and $y$ are real, then $R(x, y)=F(x, y)$. Moreover, $R$ is a polynomial which is nonvanishing on the open set

$$
\Omega_{a}=\left\{\left(z, z^{\prime}\right): \operatorname{Re} z_{i}>a,\left|\operatorname{Im} z_{i}^{\prime}\right|<a \text { for } 1 \leqq i \leqq n\right\}
$$

for each $a>0$. By Proposition 2.2 (and Remark 2 following its proof), we have for each multi-index $\mathbf{m}$ either

(i) $\partial^{\mathbf{m}} R / \partial z^{\mathbf{m}} \neq 0$ in $\Omega_{a}$

or else

(ii) $\partial^{\mathbf{m}} R / \partial z^{\mathbf{m}} \equiv 0$ in $\Omega_{a}$.

Moreover, if (ii) holds for one value of $a$ then it holds for all $a$, by analytic continuation; and $\bigcup_{a>0} \Omega_{a}$ contains the set $(0, \infty)^{n} \times \mathbb{R}^{n}$ of real points. Thus, to complete the proof for the case $f=P$, we need only determine the sign of $G_{\mathbf{m}}(x, y)$ in case (i); we use induction on each component of $\mathbf{m}$. Clearly $G_{0}(x, y) \geqq 0$. Suppose that $G_{\mathbf{m}}(x, y) \geqq 0$ for all $x \in(0, \infty)^{n}$ and all $y \in \mathbb{R}^{n}$, but that $G_{\mathbf{r}}\left(x^{\prime}, y^{\prime}\right)<0$ for some $x^{\prime} \in(0, \infty)^{n}$ and $y^{\prime} \in \mathbb{R}^{n}$, with $\mathbf{r}=\mathbf{m}+(1,0, \ldots, 0)$. Then, by the above, $G_{\mathbf{r}}(x, y)<0$ for all $x \in\left(0, \infty^{n}\right)$ and $y \in \mathbb{R}^{n}$. Fix $w=\left(x_{2}, \ldots, x_{n}\right) \in(0, \infty)^{n-1}$ and $y \in \mathbb{R}^{n}$, and consider $G_{\mathbf{m}}\left(x_{1}, w, y\right)$ and $G_{\mathbf{r}}\left(x_{1}, w, y\right)=\partial G_{\mathbf{m}} / \partial x_{1}$ as polynomials in $x_{1}$. Let $G_{\mathbf{m}}\left(x_{1}\right)=\sum_{k=0}^{K} c_{k} c_{1}^{k}$ with $c_{K} \neq 0$ (we suppress the dependence on $w$ and $y$, which are fixed once and for all). Clearly $c_{K}>0$, since otherwise $G_{m}\left(x_{1}\right) \rightarrow-\infty$ as $x_{1} \rightarrow+\infty$, contrary to the hypothesis on $G_{\mathbf{m}}$. Therefore either $G_{\mathbf{r}}\left(x_{1}\right) \equiv 0$ for all $x_{1}$ (if $K=0$ ) or else $G_{\mathbf{r}}\left(x_{1}\right)>0$ for $x_{1} \rightarrow+\infty$. But either possibility contradicts $G_{\mathbf{r}}(x, y)<0$ for all $x \in(0, \infty)^{n}$ and $y \in \mathbb{R}^{n}$. This completes the proof in the special case $f=P$.

Now let $f=\lim _{j \rightarrow \infty} P_{j}$ with each $P_{j} \in \mathscr{P}^{n}\left(D^{n}\right)$. Form $R_{j}$ from $P_{j}$ as before; since the convergence is uniform on compacts, all derivatives converge as well, so we have

$$
G_{\mathbf{m}}(x, y)=\left.\lim _{j \rightarrow \infty} \partial_{z}^{\mathbf{m}} R_{j}\left(z, z^{\prime}\right)\right|_{z=x, z^{\prime}=y}
$$

for each multi-index $\mathbf{m}$. Fix $\mathbf{m}$. Then, by the above, each $\partial_{z}^{\mathbf{m}} R_{j}$ is either strictly positive on $\bigcup_{a>0} \Omega_{a}$ or else identically zero there. It is then easy to see, using Hurwitz' 
theorem and perhaps passing to a subsequence, that $\lim _{j \rightarrow \infty} \partial_{z}^{\mathbf{m}} R_{j}$ is either strictly positive on the connected open set $\bigcup_{a>0} \Omega_{a}$ or else is identically zero there. This completes the proof.

Remarks. 1 . If $G_{\mathbf{m}} \equiv 0$ for some $\mathbf{m}$, then obviously $G_{\mathbf{m}^{\prime}} \equiv 0$ for all $\mathbf{m}^{\prime} \geqq \mathbf{m} ;$ moreover, $F(x, y)$ must be a polynomial in $x$ of degree less than $\mathbf{m}$ (with coefficients depending on $y$ ). This happens, of course, if $f$ is a polynomial; but it also happens in other cases, e.g. $f(x)=\exp (i z)$ with $n=1, m=1$.

2. If $f$ is the Laplace transform of an even, positive measure $\mu$ not supported at the origin, then case (ii) of the proposition cannot occur for any multi-index $\mathbf{m}$, since $F(x, 0)$ must increase at least exponentially rapidly as $x \rightarrow \infty$ in a suitable direction in $(0, \infty)^{n}$. This observation, combined with our form of the Lee-Yang theorem, will immediately imply (2.12), Newman's strong Lee-Yang theorem.

\section{One-Component Models}

The proof of a very general Lee-Yang Theorem for one-component ferromagnets is now essentially complete; all we have to do is to collect the pieces from the preceding section.

Definition 3.1. A finite (positive) measure $\mu$ on $\mathbb{R}^{n}(\mu \neq \equiv)$ is said to have the LeeYang property (with falloff $\beta$ ) if $\mu \in \mathscr{T}_{\beta}^{n}$ and $\hat{\mu} \in \overline{\mathscr{P}}_{1 / 4 \beta+}^{n}\left(D^{n}\right)$.

Since $\mu \neq 0$ implies that $\hat{\mu} \neq \equiv 0$, it follows that $\hat{\mu}(z) \neq 0$ for $\operatorname{Re} z>0$; this is the usual conclusion of the Lee-Yang theorem. Note, however, that $\hat{\mu} \in \overline{\mathscr{P}_{1 / 4 \beta+}^{n}}\left(D^{n}\right)$ is a stronger hypothesis: it says not only that $\hat{\mu}$ is nonvanishing in $D^{n}$ but that it is approximable by polynomials with this property.

Theorem 3.2. Let $\mu_{0}$ have the Lee-Yang property with falloff $\beta$; and let $f \in \overline{P P}_{\alpha+}^{n}\left(D^{n}\right)[\alpha<\beta]$ be nonnegative on the support of $\mu_{0}$, and strictly positive on a set of nonzero $\mu_{0}$-measure. Then $\mu=f \mu_{0}$ has the Lee-Yang property with falloff $\gamma$, for every $\gamma<\beta-\alpha[$ and $\gamma=\infty$ if $\beta=\infty]$. In particular, we can take

$$
f(\varphi)=\exp \left[\sum_{i, j=1}^{n} J_{i j} \varphi_{i} \varphi_{j}\right]
$$

with all $J_{i j} \geqq 0$, provided that $\alpha=\|J\|<\beta$. (Here $\|J\|$ is the norm of $J$ considered as a bilinear form on $\mathbb{R}^{n}$ equipped with the Euclidean norm.)

Theorem 3.2 follows immediately from Propositions 2.9 and 2.7; the positivity conditions on $f$ are needed only to ensure that $\mu \geqq 0$ and $\mu \neq \equiv$.

Corollary 3.3. Let $\left\{v_{i}\right\}_{1 \leqq i \leqq n}$ be measures on $\mathbb{R}^{1}$, each having the Lee-Yang property with falloff $\beta$; and let $J$ be a symmetric $n \times n$ matrix with nonnegative entries $[\|J\|<\beta]$. Then the measure $\mu$ on $\mathbb{R}^{n}$ given by

$$
d \mu(\varphi)=\exp \left[\sum_{i, j=1}^{n} J_{i j} \varphi_{i} \varphi_{j}\right] \prod_{i=1}^{n} d v_{i}\left(\varphi_{i}\right)
$$

has the Lee-Yang property with falloff $\gamma$, for every $\gamma<\beta-\|J\|[$ and $\gamma=\infty$ if 
$\beta=\infty]$. In particular, we can let each $v_{i}$ be an even measure in $\mathscr{T}_{\beta}^{1}$ satisfying condition (1.3).

Proof. Only the last sentence (which is Newman's [15] Lee-Yang Theorem) requires further explanation; it is a consequence of the following lemma:

Lemma 3.4. Let $v$ be an even measure in $\mathscr{T}_{\beta}^{1}$ satisfying condition (1.3). Then $v \in \overline{\mathscr{P}}_{1 / 4 \beta+}^{1}\left(D^{1}\right)$, and

$$
\hat{v}(h)=\mathrm{K} e^{b h^{2}} \prod_{j}\left(1+\frac{h^{2}}{\alpha_{j}^{2}}\right)
$$

with $K>0,0 \leqq b \leqq 1 / 4 \beta$ and $0<\alpha_{1} \leqq \alpha_{2} \leqq \ldots$, with $\sum_{j} \alpha_{j}^{-2}<\infty$; here the sequence $\left\{\alpha_{j}\right\}$ may be empty, finite or infinite.

Proof $[15,5]$. Since $\hat{v} \in \mathscr{T}_{\beta}^{1}, \hat{v}$ is an entire function either of order strictly less than 2 , or of order 2 and type at most $1 / 4 \beta$. Moreover, $\hat{v}$ is even and has only pure imaginary zeros, which we shall denote $\pm i \alpha_{j}$. If $\hat{v}$ is of order $\rho<2$, then (3.2) [with $b=0]$ follows from the Hadamard factorization theorem [33, Sect. I.10] after grouping conjugate pairs of factors. If $\hat{v}$ is of order 2 and finite type, then (3.2) follows similarly from Lindelof's extension of the Hadamard factorization Theorem [33, Sect. I.11] ; we must have $b$ real, since $\hat{v}(h)$ is real for $h$ real, and we must have $b \geqq 0$, since otherwise $\hat{v}(h)$ would vanish as $h \rightarrow \pm \infty$, which is impossible for the Laplace transform of a measure. It easily follows [28] from (3.2) that $\hat{v} \in \overline{\mathscr{P}_{1 / 4 \beta+}^{1}}\left(D^{1}\right)$, since the obvious approximating polynomials form a bounded sequence in $\mathscr{A}_{1 / 4 \beta+}^{1}$ [they are all bounded in absolute value by $\left.\hat{v}(|h|)\right]$ which is pointwise convergent to $\hat{v}$. QED.

Remarks. 1. For non-even measures $v$, condition (1.3) is not in general sufficient to imply $\hat{v} \in \overline{P_{1 / 4 \beta+}^{1}}\left(D^{1}\right)$. For example, consider $v=\delta_{a}$, so that $\hat{v}(h)=\exp (a h)$. Clearly this satisfies (1.3); but $\hat{v} \in \overline{\mathscr{P}}_{1 / 4 \beta+}^{1}\left(D^{1}\right)$ only if $a \geqq 0$. In general one must test whether $\hat{v}$ is of the form (2.6).

2. Lemma 3.4 may also be extended to even measures $v$ satisfying a weakened form of (1.3):

$$
\int e^{h \varphi} d v(\varphi) \neq 0 \text { for }|\operatorname{Re} h|>c,
$$

for some $c$. Then the $\alpha_{j}$ in (3.2) no longer need be real, but come in complexconjugate pairs and satisfy $\left|\operatorname{Im} \alpha_{j}\right| \leqq c$. Still, it is easy to show that $\sum_{j}\left|\alpha_{j}\right|^{-2}<\infty$. Moreover, we have $\hat{v} \in \mathscr{P}_{1 / 4 \beta+}^{1}\left(D_{c}\right)$. where $D_{c}=\{z: \operatorname{Re} z>c\}$. As an example of a model for which this extension is useful, consider the spin-1 measure

$$
v=\kappa \delta_{0}+\frac{1-\kappa}{2}\left(\delta_{1}+\delta_{-1}\right)
$$

with $0 \leqq \kappa \leqq 1$; here

$$
\hat{v}(h)=\kappa+(1-\kappa) \cosh h .
$$

For $0 \leqq \kappa \leqq \frac{1}{2}, v$ has the ordinary Lee-Yang property (1.3). But even for $\frac{1}{2}<\kappa<1, v$ satisfies (3.3) with $c=\cosh ^{-1}(\kappa /(1-\kappa))$. Hence we can apply the obvious generaliz- 
ation of Corollary 3.3 in which the region $D^{n}$ is replaced by $D_{c}^{n}$. (This is the reason for the otherwise pedantic insistence on arbitrary $c$ in Propositions 2.1 and 2.2.) The physical consequences of this theorem is that a ferromagnetic model with single-spin measure (3.4) is free of phase translations in the region $h>c$ (and by symmetry $h<-c$ ). Of course, for $\kappa>\frac{1}{2}$ there will in general be phase transitions at $h \neq 0$; indeed, at suitable temperature one expects the appearance of three distinct phase as $h$ is varied $[37,38]$.

In Appendix $B$ we compare our approach to the Lee-Yang Theorem with the Asano contraction method $[10-14,43]$, and give an "explanation" from within our own approach of why the Asano method works.

\section{Two-Component Models}

We now begin the application of our methods to Lee-Yang theorems for $N$-component classical ferromagnets $(N \geqq 2)$. First we must determine the zero-free region for the Laplace transform of the single-spin measure: this is the largest region for which one can even hope for a Lee-Yang theorem.

Proposition 4.1. Let $v \in \mathscr{T}_{\beta}^{N}$ be a rotationally invariant measure on $\mathbb{R}^{N}(N \geqq 2)$ satisfying

$$
\int e^{h \varphi(1)} d v(\varphi) \neq 0 \text { for } \operatorname{Re} h \neq 0 .
$$

Then the Laplace transform $\hat{v}$ is of the form

$$
\hat{v}(h)=F\left(\sum_{\alpha=1}^{N} h^{(\alpha)^{2}}\right)
$$

where $F$ is an entire function (of order at most 1) with only real negative zeros. More precisely,

$$
\hat{v}(\mathbf{h})=a e^{b \zeta} \prod_{j}\left(1+\frac{\zeta}{\alpha_{j}^{2}}\right)
$$

with $\zeta=\sum_{\alpha=1}^{N} h^{(\alpha)^{2}}, a>0,0 \leqq b \leqq 1 / 4 \beta$ and $0<\alpha_{1} \leqq \alpha_{2} \leqq \ldots$, with $\sum_{j} \alpha_{j}^{-2}<\infty$; here the sequence $\left\{\alpha_{j}\right\}$ may be empty, finite or infinite. Finally, $\hat{v} \in \mathscr{P}_{1 / 4 \beta+}^{N}\left(L_{N}\right)$, where

$$
L_{N}=L_{N}^{+} \bigcup L_{N}^{-}
$$

and

$$
L_{N}^{ \pm}=\left\{\mathbf{h}: \pm \operatorname{Re} h^{(1)}>\left[\sum_{\alpha=2}^{N}\left(\operatorname{Im} h^{(\alpha)}\right)^{2}\right]^{1 / 2}\right\}
$$

Remarks. 1. We indicate the components of a spin by Greek superscripts in parentheses, running from 1 to $N$; we label the spins on a lattice by lower-case Latin subscripts, running from 1 to $n$.

2. (4.1) says that the projection of $v$ onto the first coordinate has the Lee-Yang 
property as a measure on $\mathbb{R}^{1}$. It does not say that $v$ has the Lee-Yang property as a measure on $\mathbb{R}^{N}$. Indeed, (4.2) implies that $v$ cannot have the Lee-Yang property as a measure on $\mathbb{R}^{N}$ (unless $v$ is Gaussian, so that $\left\{\alpha_{j}\right\}$ is empty): for one can always find $\mathbf{h} \in \mathbb{R}^{N}$ with $\operatorname{Re} h^{(\alpha)}>0$ for all $\alpha$ such that $\zeta=\sum_{\alpha} h^{(\alpha)^{2}}$ takes an arbitrary negative real value.

Proof. By rotational invariance, $\hat{v}(\mathbf{h})$ is a function only of $\zeta=\sum h^{(\alpha)^{2}}$. By hypothesis (4.1), $\hat{v}(\mathbf{h})$ has only pure imaginary zeros in $h^{(1)}$ when $h^{(2)}=\ldots=h^{(N)}=0$. The representation (4.2) follows. Indeed, Lemma 3.4 implies the representation (4.3) Then $\hat{v} \in \overline{P_{1 / 4 \beta+}^{N}}\left(L_{N}\right)$ follows as in Lemma 3.4, as a result of the following lemma.

Lemma 4.2. Let $L_{N}$ be defined by (4.4) and (4.5) and let $\mathbf{h} \in L_{N}$. Then $\zeta=\sum_{\alpha=1}^{N} h^{(\alpha)^{2}}$ is
never real and negative Proof. Write $\mathbf{h}=\left(x^{(1)}+i y^{(1)}, \mathbf{x}+i \mathbf{y}\right)$ with $\mathbf{x}, \mathbf{y} \in \mathbb{R}^{N-1}$. Then $\operatorname{Re} \zeta=x^{(1)^{2}}-y^{(1)^{2}}+$ $|\mathbf{x}|^{2}-|\mathbf{y}|^{2}$ and $\operatorname{Im} \zeta=2\left(x^{(1)} y^{(1)}+\mathbf{x} \cdot \mathbf{y}\right)$. If $\operatorname{Im} \zeta=0$, then $|\mathbf{y}| \geqq\left|x^{(1)}\right|\left|y^{(1)}\right| /|\mathbf{x}|$ by the Schwarz inequality. But $\mathbf{h} \in L_{N}$ means that $\left|x^{(1)}\right|>|\mathbf{y}|$; hence $|\mathbf{x}|>\left|y^{(1)}\right|$. It follows that $\operatorname{Re} \zeta>0$. QED.

It is convenient, following Dunlop [20], to introduce the variable $\tilde{\mathbf{h}}=\left(h^{(1)}, i h^{(2)}, \ldots, i h^{(N)}\right.$. Then the set $\mathbf{h} \in L_{N}$ becomes the tube

$$
\operatorname{Re} \tilde{\mathbf{h}} \in \Gamma_{+} \bigcup \Gamma_{-},
$$

where

$$
\Gamma_{ \pm}=\left\{\mathbf{x}: \pm x^{(1)}>\left[\sum_{\alpha=2}^{N} x^{(\alpha)^{2}}\right]^{1 / 2}\right\}
$$

are the forward and backward light cones. Our ultimate goal is a Lee-Yang theorem for the region (1.10), that is, for the tube

$$
\operatorname{Re} \tilde{\mathbf{h}}_{i} \in \Gamma_{+} \text {for all } i \text {. }
$$

We study first the case $N=2$. This case is particularly simple because the tube (4.8) is equivalent by linear transformation to a product of half-planes. That is, introducing the new variables

$$
h^{ \pm}=2^{-1 / 2}\left(\widetilde{h}^{(1)} \pm \tilde{h}^{(2)}\right)=2^{-1 / 2}\left(h^{(1)} \pm i h^{(2)}\right)
$$

the tube (4.8) becomes

$$
\operatorname{Re} h_{i}^{+}>0, \operatorname{Re} h_{i}^{-}>0 \text { for all } i \text {. }
$$

We are then precisely in the situation studied in Sect. 2; the Lee-Yang Theorems of Sect. 3 carry over immediately. We need only note that

$$
\exp \left[h^{(1)} \varphi^{(1)}+h^{(2)} \varphi^{(2)}\right]=\exp \left[h^{+} \varphi^{-}+h^{-} \varphi^{+}\right]
$$

so that differentiation of the partition function with respect to $h^{ \pm}$brings down a factor of $\varphi^{\mp}$. Thus, a "ferromagnetic pair interaction" is an entire function of the 
form

$$
f(\varphi)=\exp \left[\sum_{i, j=1}^{n}\left(J_{i j}^{++} \varphi_{i}^{+} \varphi_{j}^{+}+J_{i j}^{+-} \varphi_{i}^{+} \varphi_{j}^{-}+J_{i j}^{-+} \varphi_{i}^{-} \varphi_{j}^{+}+J_{i j}^{--} \varphi_{i}^{-} \varphi_{j}^{-}\right)\right]
$$

with all coefficients $J_{i j}^{ \pm \pm} \geqq 0$. Rewriting this using (4.9), we find that

$$
f(\varphi)=\exp \left[\sum_{i, j=1}^{n} \sum_{\alpha, \beta=1}^{2} J_{i j}^{(\alpha \beta)} \varphi_{i}^{(\alpha)} \varphi_{j}^{(\beta)}\right]
$$

subject to the conditions [20]

$$
\left\{\begin{array}{l}
J_{i j}^{(11)} \text { and } J_{i j}^{(22)} \text { are real } \\
J_{i j}^{(12)} \text { and } J_{i j}^{(21)} \text { are pure imaginary } \\
J_{i j}^{(11)}+J_{i j}^{(22)} \geqq\left|J_{i j}^{(12)}-J_{i j}^{(21)}\right| \\
J_{i j}^{(11)}-J_{i j}^{(22)} \geqq\left|J_{i j}^{(12)}+J_{i j}^{(21)}\right|
\end{array}\right.
$$

for all $i, j$. In particular, in the usual case in which

$$
J_{i j}^{(12)}=J_{i j}^{(21)}=0 \text { for all } i, j,
$$

we recover the well-known [21] condition

$$
J_{i j}^{(11)} \geqq\left|J_{i j}^{(22)}\right| \text { for all } i, j \text {. }
$$

We then have the following immediate analogues of Theorem 3.2 and Corrollary 3.3 :

Theorem 4.3. Let $\mu_{0}$ be a finite (positive) measure on $\mathbb{R}^{2 n}\left(\equiv\left(\mathbb{R}^{2}\right)^{n}\right)$ with $\mu_{0} \neq \equiv$, $\mu_{0} \in \mathscr{T}_{\beta}^{2 n}$ and $\left.\hat{\mu}_{0} \in \overline{\mathscr{P}_{1 / 4 \beta+}^{2 n}}\left(L_{2}^{+}\right)^{n}\right)$; and let $f \in \overline{\mathscr{P}_{\alpha+}^{2 n}}\left(\left(L_{2}^{+}\right)^{n}\right)[\alpha<\beta]$ be nonnegative on the support of $\mu_{0}$, and strictly positive on a set of nonzero $\mu_{0}$-measure. Then $\mu=f \mu_{0}$ is a finite (positive) measure with $\mu \neq \equiv, \mu \in \mathscr{T}_{\gamma}^{2 n}$ and $\hat{\mu} \in \overline{\mathscr{P}}_{1 / 4 \gamma+}^{2 n}\left(\left(L_{2}^{+}\right)^{n}\right)$, for every $\gamma<\beta-\alpha$ $[$ and $\gamma=\infty$ if $\beta=\infty]$. In particular, we can take fof the form $(4.13)-(4.16)$, provided that $\alpha=\|J\|<\beta$. (Here $\|J\|$ is the norm of $J$ considered as a bilinear form on $\mathbb{R}^{2 n}$ equipped with the Euclidean norm.)

Corollary 4.4. For $1 \leqq i \leqq n$, let $v_{i} \in \mathscr{T}_{\beta}^{2}$ be a rotationally invariant measure on $\mathbb{R}^{2}$ satisfying condition (4.1); and let $J$ be a symmetric real $2 n \times 2 n$ matrix satisfying (4.5) - (4.16) $[\|J\|<\beta]$. Then the measure $\mu$ on $\mathbb{R}^{2 n}$ given by

$$
d \mu(\varphi)=\exp \left[\sum_{i, j=1}^{n} \sum_{\alpha=1}^{2} J_{i j}^{(\alpha)} \varphi_{i}^{(\alpha)} \varphi_{j}^{(\alpha)}\right] \prod_{i=1}^{n} d v_{i}\left(\varphi_{i}\right)
$$

has $\mu \neq \equiv, \mu \in \mathscr{T}_{\gamma}^{2 n}$ and $\hat{\mu} \in \mathscr{P P}_{1 / 4 \gamma+}^{2 n}\left(\left(L_{2}^{+}\right)^{n}\right)$, for every $\gamma<\beta-\|J\|[$ and $\gamma=\infty$ if $\beta=\infty]$. In particular, the partition function

$$
\hat{\mu}(\mathbf{h})=\int \exp \left[\sum_{i=1}^{n} \sum_{\alpha=1}^{2} h_{i}^{(\alpha)} \varphi_{i}^{(\alpha)}\right] d \mu(\varphi)
$$

is nonvanishing if

$$
\operatorname{Re} h_{i}^{(1)}>\left|\operatorname{Im} h_{i}^{(2)}\right| \text { for all } i \text {. }
$$


Remarks. 1. The last sentence of Corollary 4.4 has been proven by Dunlop [20] for the special case of the plane rotator (1.9), by quite different methods. His proof also extends [21] to the two-component $|\varphi|^{4}$ model.

2. The zero-free region obtained in Corollary 4.4 can in some cases be extended by exploiting the covariance (or invariance) of the partition function under (complex) rotations [20, Theorems 1 and 3]. For example, if the interaction is isotropic $\left(J_{i j}^{(11)}=J_{i j}^{(22)}\right.$ for all $\left.i, j\right)$, then the partition function is invariant under the simultaneous rotation of all spins; hence the region (4.19) can be extended to

$$
\bigcup_{\substack{\mathbf{u} \in \mathbb{R}^{2} \\|\mathbf{u}|=1}}\left\{\mathbf{h}: \mathbf{u} \cdot \operatorname{Re} \mathbf{h}_{i}>\left|\mathbf{u} \times \operatorname{Im} \mathbf{h}_{i}\right| \text { for all } i\right\}
$$

\section{5. $\mathbf{N}$-Component Models ( $\mathrm{N} \geqq 3$ )}

The main result of this section is Corollary 5.5, a Lee-Yang Theorem for N-component ferromagnets in which the interaction is sufficiently anisotropic [see (1.11)]. As explained in the Introduction, this result - unlike those in the previous sections -is not "best possible". But it is the only Lee-Yang Theorem we know of, for $N>3$ ! Moreover, we believe that our methods can probably be extended to derive a "best possible" Lee-Yang theorem for general $N$.

The case $N \geqq 3$ is considerably more difficult than the case $N=2$, because the tube (4.8) is no longer equivalent to a product of half-planes. The trouble is that, in three or more dimensions, the light cone is round! As a result, our fundamental theorems - Propositions 2.2 and 2.7-are no longer adequate. Rather, we require generalizations of these propositions to tubes more general than products of halfplanes. We state these conjectured generalizations in the form of two questions, to which we can provide at present only some partial answers.

Question 5.1. Let $\Gamma \subset \mathbb{R}^{n}$ be a closed convex cone, and let

$$
\Gamma^{*}=\left\{x \in \mathbb{R}^{n}: x \cdot y \geqq 0 \text { for all } y \in \Gamma\right\}
$$

be its dual cone. Let $R(v, w)$ and $S(z)$ be defined as in Proposition 2.2. Now assume that $R(v, w) \neq 0$ whenever $\operatorname{Re} v \in \Gamma^{*}$ and $\operatorname{Re} w \in \Gamma+c$. Does it follow that $S(z) \neq 0$ whenever $\operatorname{Re} z \in \Gamma+c$ ? If not, for which cones $\Gamma$ and which polynomials $R$ is it true?

Question 5.2. (a) Let $\Gamma_{1} \subset \mathbb{R}^{n_{1}}$ and $\Gamma_{2} \subset \mathbb{R}^{n_{2}}$ be open convex cones, and let $B$ be a (real or complex) $n_{1} \times n_{2}$ matrix. For which $B$ is the function

$$
f(w, z)=\exp \left(\sum_{i=1}^{n_{1}} \sum_{j=1}^{n_{2}} B_{i j} w_{i} z_{j}\right)
$$

approximable by polynomials nonvanishing in the set

$$
T_{\Gamma_{1}} \times T_{\Gamma_{2}}\left\{(w, z): \operatorname{Re} w \in \Gamma_{1}, \operatorname{Re} z \in \Gamma_{2}\right\} ?
$$

(b) [Restricted form] In the above, let $n_{1}=n_{2}=n$ and $\Gamma_{1}=\Gamma_{2}=\Gamma$, and let $B$ 
be symmetric. For which $B$ is the function

$$
f(z)=\exp \left(\sum_{i, j=1}^{n} B_{i j} z_{i} z_{j}\right)
$$

approximable by polynomials nonvanishing in the set

$$
T_{\Gamma}=\{z: \operatorname{Re} z \in \Gamma\} ?
$$

With regard to Question to Question 5.1, we have the following result, applicable to special polynomials $P_{i}$ :

Proposition 5.3. Assume that there exist vectors $\lambda^{(1)}, \ldots, \lambda^{(M)} \in \Gamma$ and polynomials $\tilde{P}_{i}(1 \leqq i \leqq k)$ in $M$ complex variables, such that

$$
P_{i}(v)=\tilde{P}_{i}\left(\lambda^{(1)} \cdot v, \ldots, \lambda^{(M)} \cdot v\right) \text { for all } i
$$

and such that

$$
\tilde{R}(\tilde{v}, w) \equiv \sum_{i=1}^{k} \tilde{P}_{i}(\tilde{v}) Q_{i}(w)
$$

is nonvanishing whenever $\operatorname{Re} \tilde{v} \geqq 0$ and $\operatorname{Re} w \in \Gamma+c$. Then

$$
S(z) \equiv \sum_{i=1}^{k} P_{i}(\partial / \partial z) Q_{i}(z)
$$

is nonvanishing whenever $\operatorname{Re} z \in \Gamma+c$.

To understand this proposition, note that the vectors $\lambda^{(1)}, \ldots, \lambda^{(M)} \in \Gamma$ define "supporting hyperplanes" for the cone $\Gamma^{*}$. Thus, if $\operatorname{Re} v \in \Gamma^{*}$, then $\operatorname{Re} \lambda^{(\ell)} \cdot v \geqq 0$ for $1 \leqq \ell \leqq \mathrm{M}$. Hence the hypothesis in $\widetilde{R}$ implies the usual hypothesis on $R$. In fact (and this is the weakness of the proposition), it is stronger: it implies that $R(v, w)$ is nonvanishing whenever $\operatorname{Re} w \in \Gamma+c$ and $\operatorname{Re} v \in\left\{\lambda^{(1)}, \ldots, \lambda^{(M)}\right\}^{*}$; and this latter cone is in general larger than $\Gamma^{*}$. (If $\Gamma^{*}$ is "round", it is necessarily larger.) Hence not all $R$ can be accommodated.

Examples. 1. Take $k=1$; then the hypothesis on $\widetilde{R}$ reduces simply to the requirement that $\tilde{P}(\tilde{v}) \neq 0$ whenever $\operatorname{Re} \tilde{v} \geqq 0$. But by (5.6) this implies that $P(v) \neq 0$ whenever $\operatorname{Re} v \in\left\{\lambda^{(1)}, \ldots, \lambda^{(M)}\right\}^{*}$. As an example of a polynomial $P(v)$ which is nonvanishing for $\operatorname{Re} v$ in $\Gamma^{*}$ but not in this larger set, let $\Gamma=\Gamma^{*}=\bar{\Gamma}_{+}$, the closed forward light cone in $\mathbb{R}^{n}(n \geqq 3)$; and let

$$
P(v)=1+\left(v_{1}+\alpha\right)^{2}-\sum_{j=2}^{n} v_{j}^{2}
$$

with $\alpha>0$. By Lemma 4.2, $P(v)$ is nonvanishing if $\operatorname{Re} v \in \Gamma^{*}$. But for $v$ real, the zero manifold of $P$ is a spacelike hyperboloid which is asymptotic to the cone $\Gamma^{*}$. Hence, if $\tilde{\Gamma}$ is a convex cone strictly larger than $\Gamma^{*}, P$ necessarily has a zero in $\tilde{\Gamma}$

2. Again let $\Gamma=\Gamma^{*}=\bar{\Gamma}_{+}$, and take $P$ of the special form

$$
P(v)=\prod_{j=2}^{n} \bar{P}_{j}\left(v_{1}, v_{j}\right) .
$$


Then the hypothesis of the proposition can be satisfied: take $M=2 n-2$; $\lambda^{(1)}=e^{(1)}+e^{(2)}, \lambda^{(2)}=e^{(1)}-e^{(2)}$, etc. [the $e^{(j)}(1 \leqq j \leqq n)$ are the unit vectors]; and take

$$
\tilde{P}(\tilde{v})=\prod_{j=2}^{n} \bar{P}_{j}\left(\frac{\tilde{v}_{2 j-3}+\tilde{v}_{2 j-2}}{2}, \frac{\tilde{v}_{2 j-3}-\tilde{v}_{2 j-2}}{2}\right) .
$$

But this is possible because the special form (5.10) implies that $P(v)$ is nonvanishing not only for $\operatorname{Re} v \in \Gamma^{*}$ but also for $\operatorname{Re} v$ in the larger cone

$$
\tilde{\Gamma}=\left\{\lambda^{(1)}, \ldots, \lambda^{(M)}\right\}^{*}=\left\{x: x_{1} \geqq \max _{2 \leqq j \leqq n}\left|x_{j}\right|\right\} .
$$

Proof of Proposition 5.3. Introduce also the new variables $\tilde{w}^{(1)}, \ldots, \tilde{w}^{(M)} \in \mathbb{C}$, and define

$$
\hat{R}(\tilde{v}, \tilde{w}, w)=\sum_{i=1}^{k} \tilde{P}_{i}(\tilde{v}) Q_{i}\left(w+\sum_{\ell=1}^{M} \tilde{w}^{(\ell)} \lambda^{(\ell)}\right) .
$$

Now $\hat{R}$ is a polynomial which is nonvanishing whenever $\operatorname{Re} \tilde{v} \geqq 0, \operatorname{Re} \tilde{w} \geqq 0$ and $\operatorname{Re} w \in \Gamma+c$. So fix $w$ with $\operatorname{Re} w \in \Gamma+c$, and apply Proposition 2.2. It follows that

$$
\hat{S}(\tilde{w}, w)=\sum_{i=1}^{k} \tilde{P}_{i}(\partial / \partial \tilde{w}) Q_{i}\left(w+\sum_{\ell=1}^{M} \tilde{w}^{(\ell)} \lambda^{(\ell)}\right)
$$

is nonvanishing whenever $\operatorname{Re} \tilde{w} \geqq 0$ and $\operatorname{Re} w \in \Gamma+c$. But

$$
\begin{aligned}
& \tilde{P}_{i}(\partial / \partial \tilde{w}) Q_{i}\left(w+\sum_{\ell=1}^{M} \tilde{w}^{(\ell)} \lambda^{(\ell)}\right) \\
& \quad=\tilde{P}_{i}\left(\lambda^{(1)} \cdot \frac{\partial}{\partial w}, \ldots, \lambda^{(M)} \cdot \frac{\partial}{\partial w}\right) Q_{i}\left(w+\sum_{\ell=1}^{M} \tilde{w}^{(\ell)} \lambda^{(\ell)}\right) \\
& \quad=P_{i}(\partial / \partial w) Q_{i}\left(w+\sum_{\ell=1}^{M} \tilde{w}^{(\ell)} \lambda^{(\ell)}\right)
\end{aligned}
$$

by (5.6). Setting $\tilde{w}=0$ completes the proof.

Remark. There is also an analogue here of Proposition 2.2(b); its proof is essentially identical.

With regard to Question 5.2, we are able to make only a few remarks:

1. We state Question 5.2. in two forms - the general form and the "on-diagonal" form - because here, unlike in Proposition 2.7, the two forms are not obviously equivalent. (They may be nonobviously equivalent.)

2. A necessary condition in case (a) is that

$$
\sum_{i=1}^{n_{1}} \sum_{j=1}^{n_{2}} B_{i j} u_{i} x_{j} \geqq 0 \text { for all } u \in \Gamma_{1}, x \in \Gamma_{2} .
$$

This follows by taking $w=\alpha u, z=\alpha x$ with $\alpha \in \mathbb{C}$ and applying the $n=1$ case of Proposition 2.7. Perhaps (5.16) is also a sufficient condition. This is certainly the case if $\Gamma_{1}$ and $\Gamma_{2}$ are the "positive hyperoctants"

$$
\Gamma_{1}=\left\{x \in \mathbb{R}^{n_{1}}: x_{i}>0 \text { for all } i\right\}, \text { etc. }
$$


since in this case (5.16) reduces to the requirement $B_{i j} \geqq 0$ for all $i, j$, and the result is true by a minor modification of the proof of Proposition 2.7. However, we have been unable to prove this conjecture for more general $\Gamma_{1}, \Gamma_{2}$.

3. In case (a) we do have the following sufficient condition:

Proposition 5.4. Let $\mu^{(1)}, \ldots, \mu^{(M)} \in \Gamma_{1}^{*}$ and $\lambda^{(1)}, \ldots, \lambda^{(M)} \in \Gamma_{2}^{*}$. Now define

$$
P_{k}(w, z)=\prod_{\ell=1}^{M}\left(\left(1+k^{-1}\left(\mu^{(\ell)} \cdot w\right)\left(\lambda^{(\ell)} \cdot z\right)\right)^{k} .\right.
$$

Then $P_{k}$ is nonvanishing in the set (5.3), and

$$
\lim _{k \rightarrow \infty} P_{k}(w, z)=f(w, z)
$$

where $f$ is defined by (5.2) and

$$
B_{i j}=\sum_{\ell=1}^{M} \mu_{i}^{(\ell)} \lambda_{j}^{(\ell)} .
$$

Moreover, the limit (5.19) holds in the topology of $\mathscr{A}_{a+}^{n_{1}+n_{2}}$ for sufficiently large a.

Proof. Since $\Gamma_{1}$ is open, we have

$$
\Gamma_{1}^{*}=\{0\} \bigcup\left\{x: x \cdot y>0 \text { for all } y \in \Gamma_{1}\right\} .
$$

Hence, for $\operatorname{Re} w \in \Gamma_{1}$, we have either $\mu^{(\ell)} \cdot w=0$ or $\operatorname{Re} \mu^{(\ell)} \cdot w>0$; and likewise for $\lambda^{(t)} z$. It follows that $P_{k}$ is nonvanishing in the set (5.3). The limit (5.19) follows from

$$
\lim _{k \rightarrow \infty}\left(1+k^{-1} x\right)^{k}=\exp (x) .
$$

The converenge in $\mathscr{A}_{a+}^{n_{1}+n_{2}}$ is proved as in Proposition 2.7.

Note that every matrix $B$ of the form (5.20) satisfies (5.16) [as it must!]; but the converse is not in general true. For example, let $\Gamma_{1}=\Gamma_{2}=\Gamma_{+}$, the forward light cone, with $n_{1}=n_{2}=n \geqq 3$. Then for a diagonal matrix $B,(5.16)$ is equivalent to

$$
B_{11} \geqq \max _{2 \leqq j \leqq n}\left|B_{j j}\right|
$$

while (5.20) is equivalent to

$$
B_{11} \geqq \sum_{j=2}^{n}\left|B_{j j}\right|
$$

[To see that (5.22) is a necessary condition, note that (5.20) implies $\operatorname{tr}(B G) \geqq 0$ for every diagonal matrix $G$ with $G_{11}=1$ and $G_{j i}= \pm 1(2 \leqq j \leqq n)$. It is easy to see that (5.22) is a sufficient condition: it suffices to take $M=n-1$, for each $j(2 \leqq j \leqq n)$ to take

$$
\begin{aligned}
& \mu^{(j-1)}=\left|B_{j j}\right|^{1 / 2}\left(\alpha_{j} e^{(1)}+e^{(j)}\right) \\
& \lambda^{(j-1)}=\left|B_{j j}\right|^{1 / 2}\left(\alpha_{j} e^{(1)}+\operatorname{sign}\left(B_{j j}\right) e^{(j)}\right)
\end{aligned}
$$

with suitable $\alpha_{j} \geqq 1$.] 
These remarks imply a Lee-Yang theorem for $N$-component ferromagnets with highly anisotropic interactions:

Corollary 5.5. For $1 \leqq i \leqq n$, let $v_{i} \in \mathscr{T}_{\beta}^{N}$ be a rotationally invariant measure on $\mathbb{R}^{N}$ satisfying condition (4.1); and let $J_{l j}^{(\alpha)}[1 \leqq i, j \leqq n, 1 \leqq \alpha \leqq N]$ be real numbers satisfying

$$
J_{i j}^{(1)} \geqq \sum_{\alpha=1}^{N}\left|J_{i j}^{(\alpha)}\right| \text { for all } i, j .
$$

Then the measure $\mu$ on $\mathbb{R}^{N n}$ given by

$$
d \mu(\varphi)=\exp \left(\sum_{i, j=1}^{n} \sum_{\alpha=1}^{N} J_{i j}^{(\alpha)} \varphi_{i}^{(\alpha)} \varphi_{j}^{(\alpha)}\right) \prod_{i=1}^{n} d v_{i}\left(\varphi_{i}\right)
$$

has $\mu \neq \equiv, \mu \in \mathscr{T}_{\gamma}^{N n}$ and $\hat{\mu} \in \overline{\mathscr{P}_{1 / 4 \gamma+}^{N n}}\left(\left(L_{N}^{+}\right)^{n}\right)$, provided that $\beta$ is sufficiently large (how large depends on $J$ and $\gamma$ ). In particular, the partition function

$$
\hat{\mu}(\mathbf{h})=\int \exp \left(\sum_{i=1}^{n} \sum_{\alpha=1}^{N} h_{i}^{(\alpha)} \varphi_{i}^{(\alpha)}\right) d \mu(\varphi)
$$

is nonvanishing if

$$
\operatorname{Re} h_{i}^{(1)}>\left(\sum_{\alpha=1}^{N}\left(\operatorname{Im} h_{i}^{(\alpha)}\right)^{2}\right)^{1 / 2} \text { for all } i .
$$

Proof. This follows from Proposition 5.4, the above remarks, and Proposition 5.3 in the accustomed manner [note that (5.18) satisfies the hypotheses of Proposition 5.3. for essentially the same reason as (5.10)]. QED

Remarks (continued). 4. In case (b) of Question 5.2, a necessary condition [analogous to $(5.16)]$ is that

$$
\sum_{i, j=1}^{n} B_{i j} x_{i} x_{j} \geqq 0 \text { for all } x \in \Gamma .
$$

However, this is clearly not a sufficient condition: (5.28) would allow any positive definite matrix $B$, for any $\Gamma$, which clearly contradicts Proposition 2.7 . We suspect that (5.16) may be a necessary condition in case (b) as well as in case (a).

5. For the special case $\Gamma=\Gamma_{+}$, the forward light cone, (5.21) is a sufficient condition for case (b): for by a minor modification of Lemma 4.2, (5.21) implies that $\sum_{i, j=1}^{n} B_{i j} z_{i} z_{j}=\sum_{i=1}^{n} B_{i i} z_{i}^{2}$ is never real and negative, for $\operatorname{Re} z \in \Gamma$; so we can write

$$
f(z)=\exp \left(\sum_{i, j=1}^{n} B_{i j} z_{i} z_{j}\right)=\lim _{k \rightarrow \infty}\left(1+k^{-1} \sum_{i, j=1}^{n} B_{i j} z_{i} z_{j}\right)^{k},
$$

a limit of polynomials nonvanishing in $\operatorname{Re} z \in \Gamma$.

6. The preceding remark would allow us to handle rotators with isotropic interactions, for arbitrary $N$, provided that we could find a satisfactory answer to Question 5.1 in this case. This is so because we can write

$$
\exp \left[J_{i j} \varphi_{i} \cdot \varphi_{j}\right]=\exp \left[\frac{1}{2} J_{i j}\left(\varphi_{i}+\varphi_{j}\right)^{2}\right] \exp \left[-\frac{1}{2} J_{i j}\left(\varphi_{i}^{2}+\varphi_{j}^{2}\right)\right]
$$


and the last factor is a harmless constant for the plane rotator measure (1.9). The same remark applies to single-spin measures which, though not of the form (1.9), satisfy the following strong version of (4.1):

$$
\int e^{h \varphi^{(1)}-c|\varphi|^{2}} d v(\varphi) \neq 0 \text { for } \operatorname{Re} h \neq 0, \text { for all } c \geqq 0
$$

- for in this case we can absorb the last factor in (5.30) into the single-spin measure. Newman [39] (see also the remarks at the end of [40]) has found all rotationally invariant measures satisfying (5.31); aside from (1.9), they are

$$
d v(\varphi)=C|\varphi|^{2 m} \exp \left(-a|\varphi|^{4}-b|\varphi|^{2}\right) \prod_{j}\left(1+|\varphi|^{2} / \alpha_{j}^{2}\right) \exp \left(-|\varphi|^{2} / \alpha_{j}^{2}\right)
$$

with $C>0, m \geqq 0$ integral, $a \geqq 0, b$ real, and $\alpha_{j}>0$ with $\sum_{j} \alpha_{j}^{-4}<\infty$; here the sequence $\left\{\alpha_{j}\right\}$ may be empty, finite or infinite. In particular, by taking $m=0$ and $\left\{\alpha_{j}\right\}$ empty, we obtain the $N$-component $|\varphi|^{4}$ lattice field theory. Finally, note that this idea also handles some anisotropic interactions, in particular those which can be written as a product of terms each of which looks like (5.30) except that $\left(\varphi_{i}+\varphi_{j}\right)^{2}$ is replaced by $\hat{\varphi}_{i j}^{2}$, with

$$
\hat{\boldsymbol{\varphi}}_{i j}=\left(\varphi_{i}^{(1)}+\varphi_{j}^{(1)}, \varphi_{i}^{(2)} \pm \varphi_{j}^{(2)}, \ldots, \varphi_{i}^{(N)} \pm \varphi_{j}^{(N)}\right)
$$

for some sequence of \pm signs. This allows some (but not all) interactions of the form (1.12).

The moral of this rather long story is that the $N$-component Lee-Yang theorem $(N \geqq 3$ ) rides on finding a satisfactory answer to Question 5.1, for the case where $\Gamma$ is a product of forward light cones. But this we must leave as an exercise for the ambitious reader.

\section{Appendix A: Alternate Proof of Newman's Lee-Yang Theorem}

In this Appendix we shall give an alternate proof of Corollary 3.3-which is a slight generalization of Newman's [15] Lee-Yang Theorem-based on the elementary Proposition 2.1 instead of the more difficult Proposition 2.2. (Actually, we shall prove only the $\beta=\infty$ case of Corollary 3.3 ; see Remark 1 following the proof.)

Note first that since $J_{i i} \geqq 0$, we can absorb the factor $\exp \left(J_{i i} \varphi_{i}^{2}\right)$ into $d v_{i}\left(\varphi_{i}\right)$ and preserve the Lee-Yang property of the latter [15, Proposition 2.4]; this follows from the $n=1$ case of Proposition 2.7. and 2.9. Hence we can assume that $J_{i i}=0$.

The proof is now by induction on $n$. By hypothesis the theorem is true for $n=1$. So assume that it is true for $n=N-1$, that is, assume that the function $\hat{\mu}_{N-1}$ defined by

$$
\hat{\mu}_{N-1}\left(h_{1}, \ldots, h_{N-1}\right)=\int \exp \left(\sum_{i, j=1}^{N-1} J_{i j} \varphi_{i} \varphi_{j}+\sum_{i=1}^{N-1} h_{i} \varphi_{i}\right) \prod_{i=1}^{N-1} d v_{i}\left(\varphi_{i}\right)
$$

lies in $\mathscr{P}_{0+}^{N-1}\left(D^{N-1}\right)$. Now by definition of $\hat{\mu}_{N}$ (and Fubini's theorem), we have

$$
\hat{\mu}_{N}\left(h_{1}, \ldots, h_{N}\right)=\int \hat{\mu}_{N-1}\left(h_{1}+\tilde{J}_{1} \varphi_{N}, \ldots, h_{N-1}+\tilde{J}_{N-1} \varphi_{N}\right) e^{h_{N} \varphi_{N}} d v_{N}\left(\varphi_{N}\right)
$$


with

$$
\tilde{J}_{i}=J_{i N}+J_{N i}=2 J_{i N}(1 \leqq i \leqq N-1) .
$$

Now the point is that since $\tilde{J}_{i} \geqq 0$, the function

$$
g\left(\varphi_{N}\right)=\hat{\mu}_{N-1}\left(h_{1}+\tilde{J}_{1} \varphi_{N}, \ldots, h_{N-1}+\tilde{J}_{N-1} \varphi_{N}\right)
$$

lies in $\overline{\mathscr{P}_{0+}^{1}}\left(D^{1}\right)$, for each fixed $\left(h_{1}, \ldots, h_{N-1}\right) \in D^{N-1}$; and so, by the $n=1$ case of Proposition 2.9, $\hat{\mu}_{N}$ lies in $\frac{1}{\mathscr{P}_{0+}^{1}}\left(D^{1}\right)$ as a function of $h_{N}$ for each fixed $\left(h_{1}, \ldots, h_{N-1}\right) \in D^{N-1}$. Of course, this is not quite what we need to prove (though it is the essential idea of the proof). To complete the rigorous proof, let $\left\{f_{j}\right\}$ be a sequence in $\mathscr{P}^{N-1}\left(D^{N-1}\right)$ converging to $\hat{\mu}_{N-1}$ in $\mathscr{A}_{0+}^{N-1}$; that is, for each $\varepsilon>0$ we have

$$
\begin{aligned}
\left|\hat{\mu}_{N-1}(\ldots)-f_{j}(\ldots)\right| & \leqq c_{j}^{(\varepsilon)} \exp \left[\varepsilon \sum_{i=1}^{N-1}\left|h_{i}+\widetilde{J}_{i} \varphi_{N}\right|^{2}\right] \\
& \leqq c_{j}^{(\varepsilon)} \exp \left[2 \varepsilon \sum_{i=1}^{N-1}\left(\left|h_{i}\right|^{2}+\widetilde{J}_{i}^{2} \varphi_{N}^{2}\right)\right]
\end{aligned}
$$

for some sequence of constants $\left\{c_{j}^{(\varepsilon)}\right\}$ converging to zero. Inserting this into (A.2), and using the hypothesis

$$
\int \exp \left(b \varphi_{N}^{2}\right) d v_{N}(\varphi)<\infty \text { for all } b
$$

we find easily that

$$
\left|\hat{\mu}_{N}(\ldots)-\int f_{j}(\ldots) e^{h_{N} \varphi_{N}} d v_{N}\left(\varphi_{N}\right)\right| \leqq K c_{j}^{(\varepsilon)} \exp \left[2 \varepsilon \sum_{i=1}^{N}\left|h_{i}\right|^{2}\right]
$$

for some constant $K<\infty$. Hence the integral converges to $\hat{\mu}_{N}$ in $\mathscr{A}_{0+}^{N}$. But the integral equals

$$
\begin{aligned}
& f_{j}\left(h_{1}+\tilde{J} \frac{\partial}{\partial h_{N}}, \ldots, h_{N-1}+\tilde{J}_{N-1} \frac{\partial}{\partial h_{N}}\right) \int e^{h_{N} \varphi_{N}} d v_{N}\left(\varphi_{N}\right) \\
& \quad \equiv g_{j}\left(\frac{\partial}{\partial h_{N}}\right) \int e^{h_{N} \varphi_{N}} d v_{N}\left(\varphi_{N}\right)
\end{aligned}
$$

(since $f_{j}$ is a polynomial, this equality is trivial), and $g_{j} \in \mathscr{P}^{1}\left(D^{1}\right)$ whenever $\left(h_{1}, \ldots, h_{N-1}\right) \in D^{N-1}$. Now let $\left\{p_{k}\right\}$ be a sequence in $\mathscr{P}^{1}\left(D^{1}\right)$ converging to $\hat{v}_{N}$ in $\mathscr{A}_{0+}^{1}$. Then, by Proposition $2.1, g_{j}\left(\partial / \partial h_{N}\right) p_{k}\left(h_{N}\right)$ is a polynomial in $\left(h_{1}, \ldots, h_{N}\right)$ which is nonvanishing in $D^{N}$ (or else is identically zero ${ }^{1}$ ). But by Proposition 2.5 and an easy estimate, $g_{j}\left(\partial / \partial h_{N}\right) p_{k}\left(h_{N}\right)$ converges in $\mathscr{A}_{0+}^{N}$ to $($ A.8) as $k \rightarrow \infty$. Hence the function (A.8) is in $\overline{\mathscr{P}_{0+}^{N}}\left(D^{N}\right)$; so by (A.7), $\hat{\mu}_{N} \in \overline{P_{0+}^{N}\left(D^{N}\right)}$ as well. This completes the proof.

1 We use Remark 2 following Proposition 2.2 (or an equivalent argument based on the last sentence of Proposition 2.1) to ensure that $g_{j}\left(\partial / \partial h_{N}\right) P_{k}\left(h_{N}\right)$ vanishes identically in $h_{N}$ for one value of $\left(h_{1}, \ldots, h_{N-1}\right)$ only it does so for all $\left(h_{1}, \ldots, h_{N-1}\right)$ 
Remarks. 1. By keeping careful track of the rate of Gaussian falloff in the above proof, one can also handle $\beta$ finite but sufficiently large (depending on the matrix $J$ ). But the inductive structure of the proof, which treats the $n$ spins asymmetrically, is unlikely to allow the optimal result $\|J\|<\beta$ proven in Corollary 3.3.

2. The inductive idea-considering the spin $\varphi_{N}$ as a "magnetic field" acting on the spins $\varphi_{1}, \ldots, \varphi_{N-1}$ - is also the basis of the proofs of the (spin- $\frac{1}{2}$ ) Lee-Yang Theorem due to Newman [15, Theorem 3.1] and Sherman [14].

\section{Appendix B: Comparison with the Asano Contraction Method}

The present approach to the Lee-Yang theorem is based on the idea that certain functions $F$-namely, $F \in \overline{\mathscr{P}_{\alpha+}^{n}}\left(D^{n}\right)$-are "universal multipliers" for Lee-Yang measures : that is, whenever $d \mu_{0}(\varphi)$ has the Lee-Yang property, so does $F(\varphi) d \mu_{0}(\varphi)$.

The Asano contraction method $[10-14,43]$, by contrast, is based on the idea that certain measures $\mu_{0}$ have the following property: if $F_{1}(\varphi) d \mu_{0}(\varphi)$ and $F_{2}(\varphi) d \mu_{0}(\varphi)$ have the Lee-Yang property, then so does $F_{1}(\varphi) F_{2}(\varphi) d \mu_{0}(\varphi)$. This idea is extremely powerful, since it allows one to prove the Lee-Yang theorem for a large model simply by verifying it for each elementary interaction, and this is often a trivial computation. Unfortunately, however, the only base-measure $\mu_{0}$ for which this idea is known to work is the uncoupled spin- $\frac{1}{2}$ Ising measure

$$
d \mu_{0}(\varphi)=\prod_{i=1}^{n} \frac{1}{2}\left[\delta\left(\varphi_{i}-a_{i}\right)+\delta\left(\varphi_{i}+a_{i}\right)\right] d \varphi_{i} .
$$

That the Asano property is not a general property of Lee-Yang measures can be seen from two simple examples in $n=1$ :

1. Let $\mu_{0}$ be the usual spin-1 measure

$$
\mu_{0}=\frac{1}{3}\left[\delta_{-1}+\delta_{0}+\delta_{1}\right] \text {. }
$$

Then $\exp \left(-b \varphi^{2}\right) d \mu_{0}(\varphi)$ has the Lee-Yang property if and only if $b \leqq \log 2$. So take $F_{1}(\varphi)=F_{2}(\varphi)=\exp \left(-b \varphi^{2}\right)$ with $\frac{1}{2} \log 2<b \leqq \log 2$; then the Asano property fails.

2. Let $\mu_{0}$ be a spin- $\frac{1}{2}$ Ising measure in a positive magnetic field:

$$
d \mu_{0}(\varphi)=e^{h_{0} \varphi}[\delta(\varphi-1)+\delta(\varphi+1)] d \varphi
$$

with $h_{0}>0$. Now let $F_{1}(\varphi)=F_{2}(\varphi)=\exp \left(-h_{1} \varphi\right)$ with $h_{0} / 2<h_{1} \leqq h_{0}$; the Asano property again fails.

On the other hand, we can "explain" in terms of our own approach why the Asano property does hold for the measure (B.1): the point is that if $F_{1}(\varphi) d \mu_{0}(\varphi)$ has the Lee-Yang property, then there exists a function $P_{1}(\varphi)$, equal to $F_{1}(\varphi)$ on the support of $\mu_{0}$, which is a universal multiplier [in fact, $\left.P_{1} \in \mathscr{P}^{n}\left(D^{n}\right)\right]$; hence

$$
F_{1}(\varphi) F_{2}(\varphi) d \mu_{0}(\varphi)=P_{1}(\varphi) F_{2}(\varphi) d \mu_{0}(\varphi)
$$

has the Lee-Yang property. To be explicit:

Proposition B.1. Let $F\left(\sigma_{1}, \ldots, \sigma_{n}\right)$ be defined for $\left\{\sigma_{1}, \ldots, \sigma_{n}\right\}= \pm 1$, and let $P\left(\varphi_{1}, \ldots, \varphi_{n}\right)$ be the unique polynomial of degree at most 1 in each variable which 
coincides with F, i.e.

$$
P\left(\varphi_{1}, \ldots, \varphi_{n}\right)=\sum_{\sigma_{1}= \pm 1} \ldots \sum_{\sigma_{n}= \pm 1} F\left(\sigma_{1}, \ldots, \sigma_{n}\right) \prod_{i=1}^{n} \frac{1+\sigma_{1} \varphi_{i}}{2} .
$$

Then the following are equivalent:

$$
\text { (a) } Q\left(z_{1}, \ldots, z_{n}\right) \equiv \sum_{\sigma_{1}= \pm 1} \ldots \sum_{\sigma_{n}= \pm 1} F\left(\sigma_{1}, \ldots, \sigma_{n}\right) \prod_{i=1}^{n} z_{i}^{(1 / 2)\left(1-\sigma_{i}\right)}
$$

is nonvanishing if all $\left|z_{i}\right|<1$ [ this is the Lee-Yang property in the activity variables $\left.z_{i}=\exp \left(-2 h_{i}\right)\right]$

(b) $P\left(\varphi_{1}, \ldots, \varphi_{n}\right)$ is nonvanishing if all $\operatorname{Re} \varphi_{i}>0$.

Proof. The transformation

$$
z_{i}=\left(1-\varphi_{i}\right) /\left(1+\varphi_{i}\right)
$$

maps $\left|z_{i}\right|<1$ onto $\operatorname{Re} \varphi_{i}>0$; and moreover

$$
P\left(\varphi_{1}, \ldots, \varphi_{n}\right)=Q\left(z_{1}, \ldots, z_{n}\right) \prod_{i=1}^{n} \frac{1+\varphi_{i}}{2} .
$$

Since $\left(1+\varphi_{i}\right) / 2 \neq 0$ for $\operatorname{Re} \varphi_{i}>0$, this completes the proof.

Remarks. 1. Although example 1 above shows that the Asano property does not hold for the spin-1 measure, a modified Asano property does hold [27] for spin 1 and in fact for all the classical discrete spins: there is a weight function $G(\varphi)$ [certain binomial coefficients] such that if $F_{1}(\varphi) d \mu_{0}(\varphi)$ and $F_{2}(\varphi) d \mu_{0}(\varphi)$ have the Lee-Yang property, then so does $G(\varphi) F_{1}(\varphi) F_{2}(\varphi) d \mu_{0}(\varphi)$. It would be interesting to have a deeper understanding of this phenomenon. Is there any direct generalization, for example, to classical Heisenberg spins?

2. The Asano contraction method has the advantage over the method of the present paper in that it is suited to studying zero-free regions other than half-planes (or circles in the activity variables) $[12,13]$.

Acknowledgement. One of us (A.S.) wishes to thank Barry Simon for a helpful conversation.

\section{References}

1. Ruelle, D. : Statistical mechanics-rigourous results. Chap. 5. New York: Benjamin 1969

2. Fröhlich, J. : Poetic phenomena in (two dimensional) quantum field theory: non-uniqueness of the vacuum, the solitons and all that. In: Les méthodes mathématiques de la théorie quantique des champs (1975 Marseille conference). Paris: CNRS 1976

3. Penrose, O., Lebowitz, J. L. : Commun. Math. Phys. 39 165-184 (1974)

4. Guerra, F., Rosen, L., Simon, B. : Commun. Math. Phys. 41, 19-32 (1975)

5. Newman, C. M. : Commun. Math. Phys. 41, 1-9 (1975)

6. Dunlop, F. : J. Stat. Phys. 21, 561-572 (1979)

7. Baker, G. A. Jr. : Phys. Rev. Lett. 20, 990-992 (1968)

8. Sokal, A. D. : J. Stat. Phys. 25, 25-50 (1981) 
9. Lee, T. D., Yang, C. N. : Phys. Rev. 87, 410-419 (1952)

10. Asano, T. : J. Phys. Soc. Jpn 29, 350-359 (1970)

11. Suzuki, M., Fisher, M. E. : J. Math. Phys (N. Y.) 12, 235-246 (1971)

12. Ruelle, D. : Phys. Rev. Lett. 26, 303-304, 870 (E) (1971)

13. Ruelle, D. : Commun. Math. Phys. 31, 265-277 (1973)

14. Simon, B. : The $\mathbf{P}(\varphi)_{2}$ Euclidean (quantum) field theory. Sect. IX.3. Princeton: Princeton University Press 1974

15. Newman, C. M. : Commun. Pure Appl. Math. 27, 143-159 (1974)

16. Dunlop, F. : J. Stat. Phys. 17, 215-228 (1977)

17. Sylvester, G. S. : A note on the Lee-Yang Theorem. Oklahoma State University preprint (1980)

18. Griffiths, R. B. : J. Math. Phys. 10, 1559-1565 (1969)

19. Simon, B., Griffiths, R. B. : Commun. Math. Phys. 33, 145-164 (1973)

20. Dunlop, F. : Commun. Math. Phys. 69, 81-88 (1979)

21. Dunlop, F., Newman, C. M. : Commun. Math. Phys. 44, 223-235 (1975)

22. Takagi, T. : Proc. Physico-Math. Soc. Japan 3, 175-179 (1921). The collected papers of T. Takagi Kuroda, S. (ed.) Tokyo: Iwanami Shoten Publishers 1973

23. Marden, M. : Geometry of polynomials (2nd edit). Providence: American Mathematical Society 1966

24. Dieudonné, J. : C. R. Acad. Sci. Paris 199, 999-1001 (1934)

25. Grace, J. H. : Proc. Cambridge Philos. Soc. 11, 352-357 (1902)

26. Obreschkoff, N.: Verteilung und Berechung der Nullstellen reeller Polynome. Berlin: VEB Deutscher Verlag der Wissenschaften 1963

27. Millard, K. Y., Viswanathan, K. S. : J. Math. Phys. 15, 1821-1825 (1974)

28. Taylor, B. A. : Some locally convex spaces of entire functions. In: Entire functions and related parts of analysis. Korevaar, J. (ed.). Proceedings of symposia in pure mathematics, Vol. XI. Providence: American Mathematical Society 1968

29. Sikkema, P. C. : Differential operators and differential equations of infinite order with constant coefficients. Groningen-Djakarta: Noordhoff 1953

30. Hille, E. : Analytic function theory, Vol. II. Boston: Ginn 1962

31. Pólya, G. : Sur les opérations fonctionelles linéaires, échangeables avec la dérivation et sur les zéros des polynômes. C. R. Acad. Sci. (Paris) 183, 413-414 (1926). Collected papers, Vol. II Boas, R. P. (ed.). pp. 261-262, 427. Cambridge: MIT Press 1974

32. Benz, E. : Comments. Math. Helv. 7, 243-289 (1935)

33. Levin, B. Ja: Distribution of zeros of entire functions. Providence: American Mathematical Society 1964 (Translated from Russian)

34. Obrechkoff, N. : Quelques classes de fonctions entières limites de polynômes et de fonctions méromorphes limites de fractions rationelles. Jn: Actualités Sci. et Ind. Vol. 891 pp. 24-25. Paris: Hermann 1941

35. Korevaar, J. : Limits of polynomials whose zeros lie in a given set. In: Entire functions and related parts of analysis (Korevaar, J. (ed.). Proceedings of symposia in pure mathematics, Vol. XI. Providence: American Mathematical Society 1968

36. Schwartz, L: Théorie des distributions (nouv. éd.). Paris: Hermann 1966

37. Pirogov, S. A., Sinai, Ya. G. : Teor. Mat. Fiz. 25, 358-369 (1975); 26, 61-76 (1976) Theor. Math. Phys. (USSR) 25, 1185-1192 (1975); 26, 39-49 (1976)

38. Gawedzki, K. : Commun. Math. Phys. 59, 117-142 (1978)

39. Newman, C. M. : Proc. Am. Math. Soc. 61, 245-251 (1976)

40. Newman, C. M. : J. Stat. Phys. 15, 399-406 (1976)

41. Heilmann, O. J., Lieb, E. H. : Commun. Math. Phys. 25, 190-232 (1972); Erratum 27, 166 (1972)

42. Dunlop, F. : Zeros of the partition function for some generalized ising models. Esztergom Colloquium. IHES Preprint 1979

43. Runnels, L. K., Lebowitz, J. L. : J. Stat. Phys. 23, 1-10 (1980)

Communicated by A. Jaffe

Received October 27, 1980 
